\title{
Climate warming reduces gut microbiota diversity in a vertebrate ectotherm
}

\author{
Elvire Bestion $^{\mathrm{a}, \mathrm{b}, \mathrm{c}, *}$, Staffan Jacob ${ }^{\mathrm{a}, \mathrm{b}, \mathrm{d}}$, Lucie Zinger $^{\mathrm{b}, \mathrm{e}}$, Lucie Di Gesu ${ }^{\mathrm{b}}$, Murielle \\ Richard $^{\mathrm{a}}$, Joël White ${ }^{\mathrm{b}}$, Julien Cote ${ }^{\mathrm{b}}$
}

Nature Ecology and Evolution 2017, 1, 0161, DOI: 10.1038/s41559-017-0161

${ }^{a}$ CNRS UMR 5321, Station d'Ecologie Theorique et Experimentale, 09200 Moulis, France

${ }^{b}$ CNRS-Université Paul Sabatier-ENSFEA UMR 5174, EDB (Laboratoire Evolution et Diversité Biologique), 118 Route de Narbonne, Bât 4R1, 31062 Toulouse Cedex 9, France

${ }^{c}$ Environmental and Sustainability Institute, College of Life and Environmental Sciences, University of Exeter, Penryn, Cornwall TR10 9FE, UK

${ }^{d}$ Université Catholique de Louvain, Earth and Life Institute, Biodiversity Research Centre, Croix du Sud 4, L7-07-04, 1348 Louvain-la-Neuve, Belgium

e Institut de Biologie de l'École Normale Supérieure, École Normale Supérieure, Paris Sciences et Lettres Research University, CNRS UMR 8197, INSERM U1024, F-75005 Paris, France

e.bestion@exeter.ac.uk

\begin{abstract}
Climate change is now considered as the greatest threat to biodiversity and ecological networks, but its impacts on the bacterial communities associated with plant or animals remain largely unknown. Here, we studied the consequences of climate warming on gut bacterial communities of an ectotherm, the common lizard (Zootoca vivipara), using a seminatural experimental approach. We found that $+2 / 3^{\circ} \mathrm{C}$ warmer climates cause a $34 \%$ loss of populations' microbiota diversity, with possible negative consequences for host survival.
\end{abstract}

Keywords: climate change - microbiome - biodiversity - host-microbiota interactions ectotherms - lizards 
Climate change is a major threat to biodiversity and ecosystem functioning ${ }^{1,2}$. It leads to phenological and range shifts ${ }^{2}$ and population and species extinctions ${ }^{2,3}$, and should hence alter many interspecific interactions. In particular species with strong, obligate interactions, such as those involving specialist predators, parasites or symbionts should be particularly impacted by changes in their interaction networks ${ }^{4}$.

One of the most intricate symbiotic relationships is probably that between animal hosts and the bacterial community inhabiting their guts ${ }^{5}$. The gut microbiota is shaped by host traits (e.g. immunity ${ }^{6}$, metabolism $^{7}$ ) and environment ${ }^{8}$ (e.g. population density or $\operatorname{diet}^{9-11}$ ) and, in turn, plays multiple essential functions for hosts, including digestion, immunity or lifehistory $^{5,12}$. Consequently, changes in the gut microbiota could lead to potential dysbioses with strong consequences for hosts and ecosystems ${ }^{13}$. These changes may be caused by rising temperatures and lead to an increase of diseases prevalence, as suggested for humans ${ }^{14}$. Understanding the factors affecting host-microbiota interactions would improve predictions on biodiversity responses to warming ${ }^{15}$. While studying such interactions in situ remains a complex challenge, a promising alternative lies in warming experiments in settings that mimic natural environments.

Here, we experimentally tested the impact of climate warming on gut bacterial microbiota of the widespread lizard species, Zootoca vivipara. We used a system composed of large seminatural enclosures in which climate can be manipulated ${ }^{3}$. In a first experiment, nine lizard populations were allocated to three climatic treatments throughout the summer: 'present climate' (current local climate), 'intermediate climate' $\left(+2^{\circ} \mathrm{C}\right)$, and 'warm climate' $\left(+3^{\circ} \mathrm{C}\right)$ which are consistent with the Intergovernmental Panel on Climate Change projections ${ }^{16}$ and critical for the life history and population dynamics of lizards ${ }^{3}$. After summer climatic treatments (mid-June to mid-September, see methods), individuals were all maintained in present climatic conditions and their cloacal bacterial communities were sampled as a proxy of hindgut the next spring.

We found warmer climates to have a strong negative effect on individual host total bacterial richness independently of bacterial abundance (Fig. 1a, Tables S1-4), with a particular impact on the most diverse bacterial phyla (Fig. 1b, Tables S5-7). This effect remained after controlling for variation in the number of sequences (Tables S1-S3, Fig. S1-4) and removing individuals with low numbers of sequences (Table S3). However, bacterial community composition was only weakly affected by climatic conditions (Fig. 1c), with no impact on community evenness (Table S8). Still, the relative abundance in major bacterial phyla differed between climates (Fig. 1d, Table S9). The relative abundance was higher in 'present climate' than in 'warm climate' for Bacteroidetes and Firmicutes. In contrast, the relative abundance of Proteobacteria tended to be higher in 'warm climate' (Fig. 1d, Table S9). We further identified 24 OTUs (Operational Taxonomic Units), members of Bacteroidetes (Bacteroidales) and Firmicutes (Lachnospiraceae), whose relative abundance significantly decreased with warming (Table S10), while 8 OTUs had their abundance increased in 'warm climate', mainly from Proteobacteria (see supplementary results). 

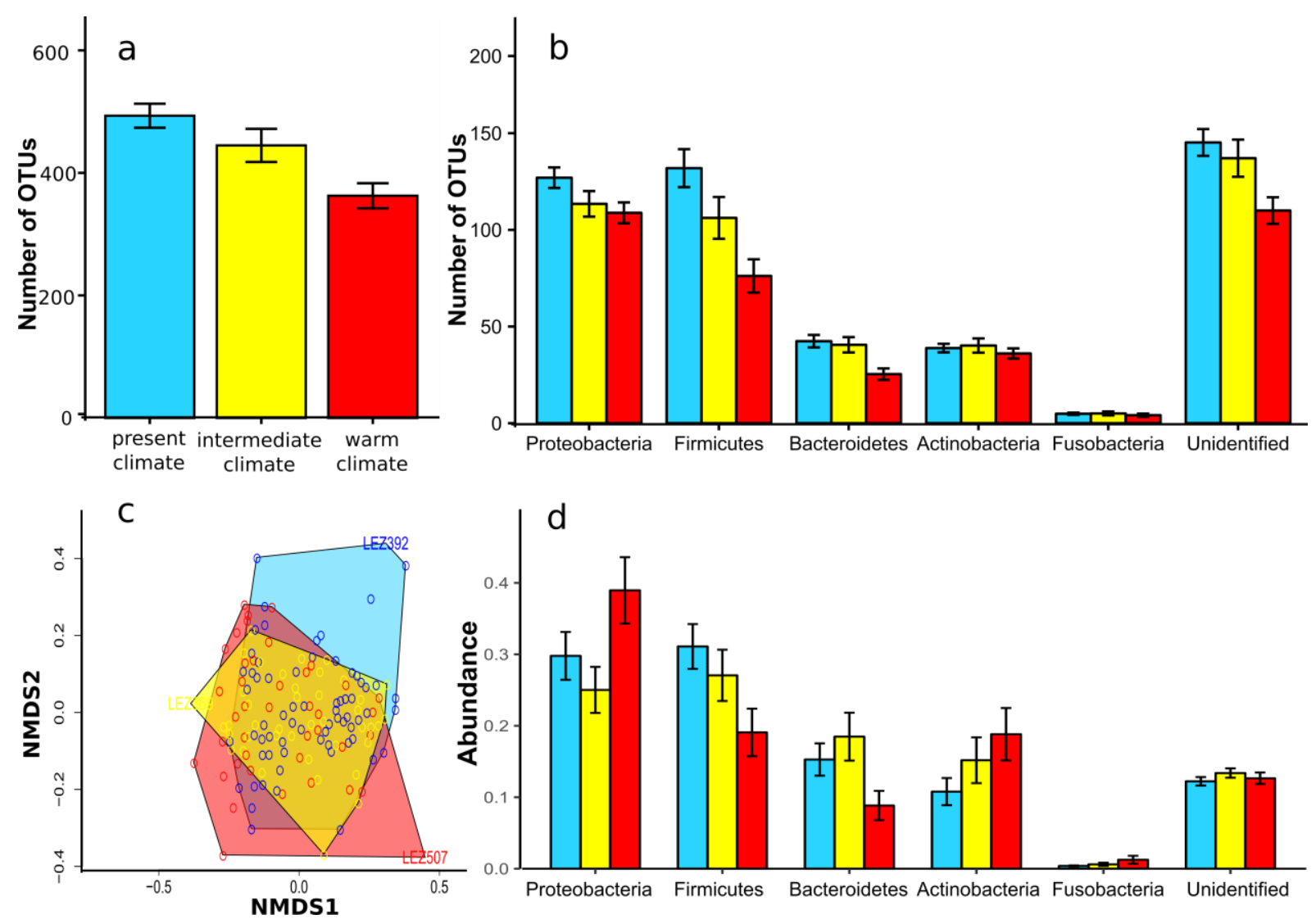

Fig. 1: Impact of climate on individual bacterial richness (mean number of taxa \pm SEM and individual points) in total (a, Table S1, p-value for climate effect: 0.017) or in the most diverse phyla (b, Table S5-7, phyla with the highest number of OTUs plus unidentified OTUs, see Table $S 7$ for significance), on hosts' community dissimilarities (c, Non-Metric Dimensional Scaling representation, PERMANOVA: $\left.F_{2,147}=1.42, p=0.01, R^{2}=0.02\right)$ and individual abundance in the most diverse phyla per climate $(d$, mean $\pm S E M$ and individual points, see Table S9 for significance). Present climate in blue ( $N=68$ lizards), intermediate in yellow $(N=41)$ and warm in red $(N=41)$. The effect of climate on bacterial richness did not rely on differences in sample sequencing depth or occurrence of rare taxa (Fig. S1-4, Table S1-4).

In addition, individual microbiota diversity loss had consequences at the population level with a 34\% decrease of total bacterial diversity with warming ('warm climate': $2451 \pm 1194$ SE, 'present climate': $3708 \pm 938 \mathrm{SE}$ ). Warming also caused greater variability in microbial community composition within host populations (mean Jaccard index within a population; 'present climate': $0.446 \pm 0.002$, 'warm climate': $0.458 \pm 0.004, \chi 2=3.86, p=0.0495)$, as was also found in a laboratory warming experiments on tadpoles ${ }^{17}$. A 3 months increase in temperature thus had long-lasting (i.e. 8 months) deleterious effects on gut bacterial diversity. This effect was also visible in our second short-term experiment, where we sampled microbiota before and after two-month long summer climatic treatments. While richness overall increased during the summer, this increase was lower in 'warm climate' due to higher bacteria extinction rate in warmer climates (Tables S11-12). Bacterial richness was therefore lower in warmer climates on the short term, congruent with the long term effects (Table S11). 
A further, unresolved question is the pathways of such climatic impacts. These impacts could arise through modification of hosts' environment (e.g. climate-induced shifts in prey community or social/sexual context affecting the pool of environmentally acquired bacteria that can be ingested or sexually/socially transmitted ${ }^{9-11}$ ) or through a change in hosts themselves (e.g. in individual condition, immune system, physiology or behaviour ${ }^{7,18}$ ). However, in the first experiment, neither host body condition, social, sexual or dietary contexts measured at the time of the microbiota sampling could explain the decrease of bacterial diversity in warmer climates (Table S13). However, these preliminary analyses cannot disentangle the different pathways of climatic impacts, such as physiological (e.g. metabolism $^{7}$ ) and behavioural mechanisms (e.g. diet composition ${ }^{11}$, mating behaviour), and should be completed by further experiments. Regardless of the exact pathway, a less diverse microbiota may affect host health and survival prospect in warmer climates.

A higher gut bacterial diversity is often beneficial to hosts ${ }^{11}$, thus a climate-driven diversity reduction might be detrimental for hosts ${ }^{19}$, particularly if it entails a loss of essential functions $^{20,21}$. Conversely, a loss of pathogenic taxa might be beneficial. The 24 OTUs notably depleted in warm climates were mainly members Bacteroidales and Lachnospiraceae, two groups particularly important in reptiles microbiota ${ }^{22,23}$. Their depletion may hence be deleterious for the host through e.g. a reduced nutrient assimilation or facilitate the colonization of the gut by pathogens, as suggested by a laboratory warming experiments on tadpoles ${ }^{17}$. To better understand these potential climate-driven changes in host-microbiota feed-backs, we studied the differences of bacterial functional profiles between warm and present climates in the first experiment through a PICRUST analysis (Tables S15-S16). Although such type of analysis is to take with caution in non-model organisms such as the common lizard, we found that functional features associated to metabolism, information processing and cellular processes were differentially abundant between climates, but in different directions for females and males (Tables S15-S16). For example, metabolismassociated functional features (e.g. lipid and energy metabolism) increased with warming in male hosts, and conversely decreased in female hosts. This difference in functional profiles was also supported by a PERMANOVA on functional features showing a significant interaction between climates and sex $\left(\mathrm{F}_{1,146}=9.35, \mathrm{p}=0.001\right)$. The functional profiles were impacted by climatic conditions in males $\left(\mathrm{F}_{1,76}=4.37, \mathrm{p}=0.018\right)$ and females $\left(\mathrm{F}_{1,70}=5.06, \mathrm{p}\right.$ $=0.004$ ), but in different ways (Fig. S4, Table S15). These contrasting functional changes in the gut microbiota shed light on interesting sex-specific responses to climatic change that will require further investigations. It is however difficult to infer the fitness consequences of such functional changes for the hosts, requiring a direct assessment of impacts on hosts.

We thus further monitored the survival of lizards involved in the first experiment in common garden enclosures in present climate for one extra year. In this third experiment, bacterial richness positively correlated with host survival the following year independently of previous climatic treatments and lizards' body condition (Table S17, Fig. S5). Host survival was further correlated to a principal component axis summarizing taxa differently abundant between climates (Table S20). The loss of bacterial taxa might therefore have a negative impact on lizard survival. As statistical models included climatic treatments and body condition, the effect of bacterial diversity is likely independent of other variables potentially modified by climatic conditions (e.g. host physiology, diet). However, since these last results are correlative, further studies involving direct manipulation of microbial diversity (e.g. through faecal transplant experiments) would be needed to fully validate our conclusion. Nonetheless, this is the first study, to our knowledge, to show that climate warming induces alterations on diversity, structure and function of a neglected level of biological diversity in a natural setting, with potential adverse consequences on hosts at both individual and 
population scales. Future studies should hence aim at investigating this overlooked effect of climate change to better predict impact on biodiversity.

\section{Methods summary}

First experiment: Effect of climate on microbiota on the long term

We used lizard populations maintained in the Metatron, an ensemble of 48 semi-natural enclosures of $100 \mathrm{~m}^{2}$, typical of lizards' habitats, in which climate can be manipulated ${ }^{3}$ (see Supplementary Methods for a full methods description, http://themetatron.weebly.com/). In June 2012, nine enclosures were allocated to three treatments, present, intermediate and warm climate $^{3} .241$ adults and yearlings and 365 juveniles were then released into nine populations (adults: $6 \pm 1 \hat{\jmath}, 10 \pm 1$, yearlings: $11 \pm 1$, juveniles: $40 \pm 3$ ).

Lizards were maintained a year in the enclosures, with climatic treatments being effective from mid-June to mid-September 2012 (mean daily temperatures, present: $26.6 \pm 0.4^{\circ} \mathrm{C}$, intermediate: $28.2 \pm 0.5^{\circ} \mathrm{C}$, and warm climate: $28.4 \pm 0.5^{\circ} \mathrm{C}$; maximum daily temperatures: present: $29.3 \pm 0.4^{\circ} \mathrm{C}$, intermediate: $31.5 \pm 0.5^{\circ} \mathrm{C}$, and warm climate: $32.1 \pm 0.6^{\circ} \mathrm{C}$, mean $\left.\pm \mathrm{SE}\right)^{3}$. In May 2013, surviving lizards (92 adults, 73 yearlings) were sampled for cloacal microbiota analyses. In reptiles, cloacal samples may encompass the breadth of bacterial diversity in the gut $^{23}$, even if it should more likely be representative of the hindgut bacterial community ${ }^{22}$. We used high-throughput sequencing of the bacterial 16S rRNA gene to quantify cloacal microbiota's diversity and composition ${ }^{24}$. Molecular, bioinformatic and statistical analyses are described in supplementary methods.

\section{Second experiment: microbiota changes on the short term}

To investigate whether climate-induced changes in bacterial communities resulted from differences in bacterial extinction rates or in the proportion of new taxa gained, we created a follow-up experiment during the summer 2013 with a set of 84 new adult individuals. After cloacal microbiota sampling in June, individuals were released into 4 present and 4 warm enclosures in mid-July (adults: $11 \hat{\jmath}, 10$ ). Populations were maintained two months in present and warm climates and surviving $(\mathrm{N}=48)$ lizards' microbiota was resampled midSeptember.

\section{Third experiment: Effect of microbiota on survival in a common garden}

In July 2013, we released lizards from the first experiment into 5 present climate enclosures to test the impact of microbiota richness at $t$ on survival at $t+1.78$ individuals (present: 37, intermediate: 20, warm: 21) were randomly assigned to an enclosure. Populations were completed with juveniles and other adults and yearlings previously maintained in separate enclosures (adults: $12 \pm 0$ ㅇ, $7 \pm 1 \hat{\jmath}$, yearlings: $7 \pm 1$, juveniles: $35 \pm 2$ ). Populations were left one year, and in May 2014, we recaptured all survivors.

\section{References}

1. Singer, A., Travis, J.M.J. \& Johst, K. Oikos 122, 358-366 (2013).

2. Parmesan, C. Annual Review of Ecology, Evolution, and Systematics 37, 637-669 (2006).

3. Bestion, E., Teyssier, A., Richard, M., Clobert, J. \& Cote, J. PLoS Biol 13, e1002281 (2015).

4. Dunn, R.R., Harris, N.C., Colwell, R.K., Koh, L.P. \& Sodhi, N.S. Proc. R. Soc. B 276, 3037-3045 (2009).

5. Gilbert, S.F., Sapp, J. \& Tauber, A.I. The Quarterly Review of Biology 87, 325-341 (2012).

6. Ruiz-Rodríguez, M. et al. Journal of Avian Biology 40, 42-48 (2009). 
7. Carey, H.V., Walters, W.A. \& Knight, R. American Journal of Physiology - Regulatory, Integrative and Comparative Physiology 304, R33-R42 (2013).

8. Sullam, K.E. et al. Molecular Ecology 21, 3363-3378 (2012).

9. Lurgi, M., López, B.C. \& Montoya, J.M. Phil. Trans. R. Soc. B 367, 3050-3057 (2012).

10. White, J., Richard, M., Massot, M. \& Meylan, S. PLoS ONE 6, e22339 (2011).

11. Bolnick, D.I. et al. Ecology Letters 17, 979-987 (2014).

12. Sison-Mangus, M.P., Mushegian, A.A. \& Ebert, D. ISME J 9, 59-67 (2015).

13. Turnbaugh, P.J. et al. Nature 449, 804-810 (2007).

14. Kim, B.-J., Lee, S.-Y., Kim, H.-B., Lee, E. \& Hong, S.-J. Allergy, Asthma \& Immunology Research 6, 389 (2014).

15. Altizer, S., Ostfeld, R.S., Johnson, P.T.J., Kutz, S. \& Harvell, C.D. Science 341, 514-519 (2013).

16. IPCC (Cambridge University Press: Cambridge, United Kingdom and New York, NY, USA, 2013).

17. Kohl, K.D. \& Yahn, J. Environ Microbiol 18, 1561-1565 (2016).

18. Dillon, M.E., Wang, G. \& Huey, R.B. Nature 467, 704-706 (2010).

19. Le Chatelier, E. et al. Nature 500, 541-546 (2013).

20. Mandrioli, M. Invertebrate Survival Journal 9, 58-63 (2012).

21. Kikuchi, Y. et al. mBio 7, e01578-16 (2016).

22. Kohl, K.D. et al. Mol Ecol 26, 1175-1189 (2017).doi:10.1111/mec.13921

23. Colston, T.J., Noonan, B.P. \& Jackson, C.R. PLOS ONE 10, e0128793 (2015).

24. Lozupone, C.A., Stombaugh, J.I., Gordon, J.I., Jansson, J.K. \& Knight, R. Nature 489, 220-230 (2012).

\section{Acknowledgements}

We thank Antoine Cave, Bastien Rémurier, Laure Geidel and Alice Fournier for fieldwork assistance and Hélène Holota for performing genetic extractions, and Jérôme Chave and Gabriel Yvon-Durocher for helpful comments on the manuscript. We are also grateful to the genotoul bioinformatics platform Toulouse Midi-Pyrenees (Bioinfo Genotoul) for providing computing and storage resources. This work has been done in the Station d'Ecologie Theorique et Experimentale (CNRS, UMR 5321) and the Laboratoire Evolution et Diversité Biologique (CNRS, UPS, ENSFEA, UMR 5174), supported by the Laboratoire d'Excellence (LABEX) TULIP (ANR -10-LABX-41) and CEBA (ANR-10-LABX-25-01). JC was supported by an ANR-12-JSV7-0004-01 and JW by an AOI Fonds Scientifique - ENFA.

\section{Authors contributions}

EB, JW and JC designed the study. EB and JC performed the experiments. EB collected genetic data, LDG and JW extracted microbial genetic data and MR extracted and analysed lizard genetic data. SJ, JW and LZ performed molecular and bioinformatics analyses, and EB, JC, LDG and SJ analysed the data. EB wrote the first draft and JC, JW, SJ and LZ significantly contributed in the writing of the manuscript.

Competing financial interests: The authors declare no conflicts of interest.

Data availability. The data presented in this manuscript are available on Pangaea.de (https://doi.pangaea.de/10.1594/PANGAEA.874404 (Elvire Bestion, University of Exeter)).

How to cite this article: Bestion, E. et al. Climate warming reduces gut microbiota diversity in a vertebrate ectotherm. Nat. Ecol. Evol. 1, 0161 (2017). 


\section{Supporting information:}

\section{Extended methods}

\section{Species and experimental system}

Lizards were captured in 2010 from natural populations of the Cévennes Mountains (France, $44^{\circ} 27^{\prime} \mathrm{N}, 3^{\circ} 44^{\prime} \mathrm{E}$, Licence no.2010-189-16 DREAL), marked by toe-clipping and translocated to the Metatron, an ensemble of semi-natural enclosures located at the Moulis field station (France, $43^{\circ} 01^{\prime} \mathrm{N}, 1^{\circ} 05^{\prime} \mathrm{E}$, see ${ }^{1-3}$ ). This structure offers 48 interconnected enclosures of $100 \mathrm{~m}^{2}$, each delimited by tarpaulins buried into the ground and enclosed by a net, preventing escape and predation. Enclosures act as mini-ecosystems typical of lizard habitats, with diverse natural vegetation, hides, rocks and invertebrate self-maintaining communities $^{2}$. Temperature, illuminance and hygrometry within enclosures are monitored continuously and can be manipulated through motor-driven shutters and sprinklers. Lizards were maintained in the Metatron for two years prior to the experiment in 'present climate' conditions (see next section), allowing acclimatization.

In May 2012, repeated capture sessions permitted capturing all surviving lizards (adults: 89 females, 51 males; yearlings: 55 females, 46 males). Lizards were kept in the laboratory according to methods described in ${ }^{2}$ until female parturition. Females gave birth to 365 viable offspring between June and July. A tail tip from every individual was collected for genetic identification.

\section{First experiment: Effect of climate on microbiota on the long term}

Nine Metatron enclosures were chosen to host 9 populations. Enclosures were divided into three groups chosen to be homogeneous respective to the plant cover and composition $\left(\mathrm{F}_{2,6}=\right.$ 1.01, $\mathrm{p}=0.42$ and $\left.\mathrm{F}_{2,6}=0.01, \mathrm{p}=0.99\right)$ and to the invertebrate abundance and composition (insect abundance: $\mathrm{F}_{2,6}=1.78, \mathrm{p}=0.25$, spider abundance: $\mathrm{F}_{2,6}=0.54, \mathrm{p}=0.61$, insect richness: $F_{2,6}=0.91, p=0.45$, spider richness: $\left.F_{2,6}=0.27, p=0.77\right)$. In June 2012, three treatments were created using automatic shutters ( $\mathrm{see}^{2}$ for details): 'present climate' (PC), 'intermediate climate' (IC) and 'warm climate' (WC). 'Present climate' enclosures corresponded to the local area climate while 'warm climate' enclosures were on average 2 and $3^{\circ} \mathrm{C}$ warmer (mean daily temperatures, PC: $26.6 \pm 0.4{ }^{\circ} \mathrm{C}$, IC: $28.2 \pm 0.5^{\circ} \mathrm{C}$, WC: $28.4 \pm$ $0.5^{\circ} \mathrm{C}$; maximum daily temperatures: PC: $29.3 \pm 0.4^{\circ} \mathrm{C}, \mathrm{IC}: 31.5 \pm 0.5^{\circ} \mathrm{C}$, WC: $32.1 \pm 0.6^{\circ} \mathrm{C}$, mean $\pm \mathrm{SE}$ ), consistent with the $3^{\circ} \mathrm{C}$ temperature increase of the IPCC projections for southern Europe by $2080^{4}$.

Early June 2012, males and yearlings were released into the enclosures. Females and their offspring were released from June to early July. Each population included $6 \pm 1$ adult males, $10 \pm 1$ adult females, $11 \pm 1$ yearlings, and $40 \pm 3$ juveniles, matching densities in natural populations ${ }^{5}$. There were no differences between treatments in a series of phenotypic traits including body size and mass (snout-vent length: $\mathrm{F}_{2,238}=0.05, \mathrm{p}=0.95$ and $\mathrm{F}_{2,362}=1.21, \mathrm{p}=$ 0.30, total length: $\mathrm{F}_{2,238}=0.04, \mathrm{p}=0.96$ and $\mathrm{F}_{2,362}=0.11, \mathrm{p}=0.90$, body mass: $\mathrm{F}_{2,238}=0.007$, $\mathrm{p}=0.99$ and $\mathrm{F}_{2,362}=0.28, \mathrm{p}=0.75$ for adults and yearlings and juveniles respectively), coloration (dorsal blackness: $\mathrm{F}_{2,238}=0.93, \mathrm{p}=0.40$, ventral coloration: $\mathrm{F}_{2,235}=0.0007, \mathrm{p}=$ 0.99 for adults and yearlings), behaviour (activity: $F_{2,237}=0.02, p=0.97$ and $F_{2,362}=0.56, p=$ 0.57, exploration: $F_{2,237}=0.20, p=0.82$ and $F_{2,362}=0.68, p=0.51$, sociability: $F_{2,237}=0.21, p$ $=0.81$ and $\mathrm{F}_{2,362}=0.56, \mathrm{p}=0.57$, preferred temperature: $\mathrm{F}_{2,237}=0.22, \mathrm{p}=0.80$ and $\mathrm{F}_{2,362}=$ $1.47, \mathrm{p}=0.23$ for adults and yearlings and juveniles respectively), adult female reproduction (reproductive status: $\mathrm{F}_{2,136}=0.30, \mathrm{p}=0.74$, clutch size: $\mathrm{F}_{2,85}=0.15, \mathrm{p}=0.86$ ) and juvenile 
birth-date $\left(\mathrm{F}_{2,362}=0.82, \mathrm{p}=0.44\right)$. The absence of differences in any measured phenotypic trait combined with the large sample size (241 adults and yearlings and 365 juveniles released) makes very unlikely the possibility of a bias in bacterial communities' richness at the beginning of the experiment. Lizards were maintained the whole year in the enclosures, with climatic treatments effective during the summer (from mid-June to mid-September) and no differences in temperature between treatments during the winter and spring ${ }^{2}$. There was no food supplementation during the experiments as the Metatron is rich in invertebrate prey ${ }^{2}$. Invertebrate diversity and abundance were high in the enclosures, with no differences between climatic treatments at the beginning and at the end of the experiment ${ }^{2}$.

In May 2013, we recaptured all surviving lizards (92 out of 241 adults and 73 out of 365 oneyear old juveniles) during $>10$ capture sessions without release, brought them into the laboratory and sampled them for cloacal microbiota analyses. The cloacal microbiota was sampled using a standardized method already used in this species ${ }^{6}$ which involves flushing the cloaca with $2 \times 45 \mu \mathrm{L}$ of a sterile saline solution (Phosphate buffer saline, $\mathrm{pH}$ 7.4, Sigma) using a sterile pipette. Samples were immediately placed in a sterile $1.5 \mathrm{ml}$ vial and stored at $20^{\circ} \mathrm{C}$. In reptiles, cloacal samples may encompass the breadth of bacterial diversity in the gut $^{7}$, even if it should more likely be representative of the hindgut bacterial community ${ }^{8}$. Lizards were then maintained in the laboratory until female parturition. At birth, a tail tip from offspring was collected for further genetic identification and parentage analysis according to methods described in ${ }^{9}$, allowing to quantify the number of sexual partners of each lizard.

\section{Second experiment: microbiota changes on the short term}

To investigate whether climate-induced changes in bacterial communities resulted from differences in bacterial extinction rates or in the proportion of new taxa gained, we performed an additional experiment during the summer 2013. A set of 84 adults captured in the Cevennes Mountains was sampled for their cloacal microbiota in June 2013. After sampling of cloacal microbiota, individuals were released in 'present' and 'warm' climate enclosures to form 8 populations of 10 females and 11 males in mid-July 2013. There were no differences between treatments in adult body size (snout-vent length: $F_{1,82}=0.0006, p=0.98$, total length $\left.\mathrm{F}_{1,82}=0.35, \mathrm{p}=0.55\right)$, body mass $\left(\mathrm{F}_{1,82}=0.08, \mathrm{p}=0.78\right)$, coloration (dorsal blackness: $\mathrm{F}_{1,82}=$ $0.14, \mathrm{p}=0.71)$ and initial bacterial richness $\left(\mathrm{F}_{1,82}=0.97, \mathrm{p}=0.33\right)$. Individuals were maintained in these climatic conditions during the summer, and in mid-September, after 2 months of climatic treatment, we captured all surviving individuals (48 individuals, 21 in present climate and 27 in warm climate) and resampled their microbiota to monitor changes in microbiota over the summer of climatic treatment.

\section{Third experiment: Effect of microbiota on survival in a common garden}

In July 2013, we released lizards from the first experiment, along with lizards from another experiment, in 5 enclosures in 'present climate' to test whether bacterial community richness measured in May 2013 impacted their survival the year after. 78 adults and yearlings were released (females: 21 adults, 16 yearlings, males: 23 adults, 18 yearlings), 37 from 'present' climate in 2012, 20 from 'intermediate' climate and 21 from 'warm' climate. Lizards were distributed among the 5 enclosures in order to have similar mixes of lizards from treatments and enclosures of the previous experiment. Enclosures were completed with juveniles and other adults and yearlings to form populations of $12 \pm 0$ adult females, $7 \pm 1$ adult males, $7 \pm$ 1 yearlings and $35 \pm 2$ juveniles. The additional lizards were previously maintained in separate enclosures for a year before this experiment. These lizards were added to obtain normal lizard densities and age structures in the enclosures, however there were not 
considered when assessing the impact of bacterial community richness on survival. This full mixing design allows studying the link between bacterial richness and survival independently of any other population and community changes induced by climatic treatments in the previous experiment. Populations were left one year, and in May 2014, repeated capture sessions allowed to capture every surviving individual $(\mathrm{N}=40)$.

\section{Molecular Analyses}

Bacterial DNA extraction was performed using a standard protocol designed for the purification of total DNA from gram-positive bacteria with the Qiagen DNeasy Blood \& Tissue Kit (Qiagen, Venlo, Netherlands). The concentration of genomic DNA extracts was $13.89 \pm 1.97 \mathrm{ng} / \mathrm{ul}$. Experimenter was blind to climatic treatments during molecular analyses.

PCR amplifications were performed in $30 \mu \mathrm{L}$ containing $3 \mu \mathrm{L}$ of diluted DNA extract. Each PCR mixture was composed of $0.25 \mu \mathrm{M}$ of each primer, 1U of AmpliTaq Gold DNA Polymerase (Applied Biosystems, Foster City, CA, USA), $2.5 \mathrm{mM}$ of $\mathrm{MgCl}_{2}, 1 \mathrm{x}$ of Taq Buffer, $0.2 \mathrm{mM}$ of each dNTP and $4 \mathrm{ng}$ of bovine serum albumin (Promega Corporation, Madison, USA). PCR conditions consisted of an initial denaturation at $95^{\circ} \mathrm{C}$ for $5 \mathrm{~min}$, followed by 35 cycles of denaturation $\left(95^{\circ} \mathrm{C}\right.$ for $\left.30 \mathrm{~s}\right)$, annealing $\left(57^{\circ} \mathrm{C}\right.$ for $\left.30 \mathrm{~s}\right)$ and elongation $\left(72^{\circ} \mathrm{C}\right.$ for $\left.30 \mathrm{~s}\right)$. The universal primer used specifically amplified the v5-6 region (ca $295 \mathrm{bp}$ length) of the bacterial 16S rRNA gene (BACTB-F: 5'GGATTAGATACCCTGGTAGT-3'; and BACTB-R: 5'-CACGACACGAGCTGACG-3 ${ }^{10}$ ). To discriminate samples after sequencing, both forward and reverse primers were labelled at the 5' end with a combination of two different 8 bp tags. PCR products were purified using the QIAquick PCR purification Kit (Qiagen GmbH, Hilden, Germany) and pooled. A library of the amplicons multiplex was prepared following the MetaFast protocol and then sequenced with an Illumina MiSeq platform using the 2x250 bp paired-end technology at Fasteris SA (Plan-les-Ouates, Switzerland). PCR blank controls were included in the sequenced multiplex to detect potential reagent contaminants.

\section{Bioinformatic analysis}

Illumina sequences data were curated as described in ${ }^{11}$ using OBITools package ${ }^{12}$ but with some adjustments. First, we aligned paired-end reads by taking into account phred qualities at each sequencing site. This, together with the fact that each paired-end read cover $>80 \%$ of the amplified fragment allowed us to retrieve full-length barcodes with an overall relatively high sequencing quality. Next, the consensus reads were assigned to their respective samples. Any consensus read presenting 0 and 2 mismatches on one tag or primer site was considered as being of low quality and excluded. We also excluded any consensus read with low assembly score, as these may correspond either to non-overlapping paired-end reads, or to low-quality paired-end reads ${ }^{12}$. We further excluded consensus reads containing ambiguous bases (i.e. "N") or shorter than 70 bp. Strictly identical reads were dereplicated, and singletons (i.e. one single occurrence over the entire dataset) were removed as these most likely correspond to artifactual sequences. To control for potential PCR/sequencing errors we used the obiclean algorithm $^{12}$ and the remaining sequences were clustered into OTUs (Operational Taxonomic Units) based on their similarity. The clustering was done with the sumaclust algorithm (https://git.metabarcoding.org/obitools/sumatra/wikis/home), with a 97\% similarity threshold. The most abundant sequences of each OTU were then taxonomically assigned using the RDPII classifier ${ }^{13}$ with the RDPII database release 11 (May 2015). We here considered a taxonomic assignment as reliable when its probability (provided at each taxonomic level) was $>0.8$. Next, we removed all OTUs with a total read abundance $<10$ reads or detected in only one sample. Finally, we used the PCR blank controls to remove potential contaminants from 
the data set (241 low-abundance OTUs removed out of 6413 OTUs). Experimenter was blind to climatic treatment during bioinfirmatic analyses.

The final dataset consisted in 424050 reads and 6654 OTUs for 150 samples. About $99.0 \%$ of the 6654 OTUs detected in the whole dataset belongs to 5 phyla plus unidentified OTUs (Unidentified taxa $=28.7 \%$, Proteobacteria $=28.1 \%$, Firmicutes $=21.9 \%$, Bacteroidetes $=$ $9.9 \%$, Actinobacteria $=9.0 \%$ and Fusobacteria $=1.3 \%$ ) while the remaining $1 \%$ corresponded to 12 other phyla (Acidobacteria, Candidatus Saccharibacteria, Verrucomicrobia, Chlamydiae, Planctomycetes, Spirochaetes, Deinococcus-Thermus, Tenericutes, Cyanobacteria, Gemmatimonadetes, Chloroflexi and Armatimonadetes).

\section{Statistical analyses}

\section{Effect of climate change on individual bacterial richness in the first experiment}

We investigated climatic impacts on total bacterial richness (i.e. total number of OTUs per individual), identified bacterial richness (i.e. number of taxonomically assigned OTUs) using linear mixed models with lmer procedure in $\mathrm{R}$ version $3.1 .1^{14,15}$.

We fitted a full model including climatic treatment as a continuous variable, plus sex and age classes ( 1 or $\geq 2$ years old) of individuals as covariates and every possible interaction, and sample sequencing depth and its interaction with climate plus enclosure identity as a random intercept. The interaction between age class and sex and climate was added because they are important modulators of individuals microbiota and because lizards have been found to respond differently to climate depending on age and $\operatorname{sex}^{2}$, therefore it is likely that it would affect bacterial microbiota differently. We also included the enclosure identity as a random intercept to account for potential differences among enclosures. The inclusion in the model of the sample sequencing depth (centred around the mean and scaled by dividing by the standard deviation) and its interaction with climatic treatment was done in order to account for potential differences in bacterial load and/or bacterial diversity sampling through sequencing (i.e. different sequencing depth among samples) between climates, although we found no significant impact of climatic conditions on sample sequencing coverage, as estimated by the Good coverage [estimator: 1-(singletons/total number of sequences), Supplementary Fig. 1a] and on sample sequencing depth (number of sequences by sample, Supplementary Fig. 1b).

We compared this full model including climate, age, sex and their interaction and climate and sequencing depth interactions plus random enclosure to all derived simpler models through AIC. When several models had close AICs $(\triangle \mathrm{AIC}<2)$, we used a model averaging method following ${ }^{16}$ with the MuMIn R package ${ }^{17}$. The same model averaging method was used for all subsequent analysis. Residuals were screened visually for normality and homoscedasticity.

We first tested the impact of climatic treatment on total bacteria richness (Supplementary Table 1). Because there were a noticeable number of unidentified OTUs, we redid this analysis on the taxonomically identified bacteria richness (Supplementary Table 2). Accumulation curves showed that the richness in each treatment was well covered, although the asymptote was not reached (Supplementary Fig. 3). We also checked that the effect of climatic conditions on bacterial richness did not rely only on a disappearance of very rare bacterial OTUs by (1) verifying that there were no significant differences among climatic treatments in sample sequencing depths and coverage (Supplementary Fig. 1), suggesting that the detection power of rare taxa was not lower in warmer climates, (2) removing host individuals for which sample sequencing depth was too low $(<1000$ reads, $12 \%$ of individuals, Supplementary Table 3), and (3) restricting our dataset to individuals with less 
than 4000 sequences to completely removed any differences in the number of sequence between climates. Finally, we (3) truncated the data with several abundance/prevalence cutoffs to check whether the effect of climatic conditions on bacterial richness did not rely only on a disappearance of very rare bacterial OTUs (Supplementary Table 4).

We then checked the impact of climatic conditions on OTUs richness of each of the most diverse phyla (5 phyla with the highest number of OTUs plus unidentified OTUs), standardized by phylum to avoid heteroscedasticity, with a repeated linear mixed model including climatic treatment, phylum identity and their interaction as fixed effects and enclosure and lizard identities as random intercepts (Supplementary Tables 5 and 6). We further investigated climatic effects on bacterial richness for each phylum. We did so with non-parametric spearman correlation models of phylum taxa richness as a function of climatic conditions as using parametric tests would lead to high non-normality of model residuals. To avoid multiple testing issues, we provide corrected p-values with the Holm-Bonferroni sequential correction and the Benjamini \& Hochberg correction (Supplementary Table 7).

\section{Effect of climate change on individual community structure in the first experiment}

We further investigated climatic effects on community structure by calculating 1) bacterial evenness (i.e. Shannon index/ln(bacterial richness), Supplementary Table 8) and 2) dissimilarity of hosts' microbial communities between climates using the Bray-Curtis index. Dissimilarities between hosts' microbial communities were then vizualized with a Non Metric Dimensional Scaling analysis (Fig. 1c). We finally tested whether community composition differed between climates by performing a PERMANOVA on the Bray-Curtis distance matrices with $\mathrm{R}$ adonis function from the vegan package (https://cran.rproject.org/web/packages/vegan/index.html), with 999 permutations (Fig. 1c).

We then further explored what bacterial taxa differed between the climatic treatments. We first described and tested, using a Kruskall-wallis test, differences in relative abundances in the 6 most diverse and more abundant bacterial groups between present, intermediate and warm climates (Supplementary Table 9). We further explored the bacterial OTUs responsible for differences in diversity and community structure between present and warm climates (Supplementary Table 10). To do so, we coupled two approaches and searched consensus between them to ascertain the differences of taxa between climates. First, we used a MannWhitney test on the relative abundance of each OTU with a 0.005 p-value threshold. Second, we used a linear discriminant analysis (LDA) effect size (LEfSe) using the Galaxy platform (http://huttenhower.sph.harvard.edu/galaxy/) with a $0.005 \mathrm{p}$-value threshold and a LDA score $>2$. While the average difference in diversity between treatments was 131 OTUs, we found 49 OTUs being significantly different between them, 17 unidentified OTUs and 32 OTUs with taxonomic information below the phylum level (Supplementary Table 10).

Finally, we also determine which functional characteristics of the bacterial communities differed amongst climatic treatments. These were inferred with the PICRUSt program ${ }^{18}$ using the Greengenes database (v13.5) with a 97\% threshold to perform a taxonomic assignment and the online Galaxy platform to perform copy number normalisation of each OTU as recommended in ${ }^{18}$. We also conducted a metagenome prediction of each sample, from which main functions, as categorized into KEGG pathways ${ }^{19}$, were retrieved. This resulted in a table with the KEGG pathway abundances for each sample. We choose to focus on the 4 functional groups ((KEGG-Level 1 functional categories, Metabolism, Environmental Information Processing, Genetic Information Processing, Cellular Processes) that are relevant for bacteria $^{18}$. These predictions depend on whether the bacterial taxa present are found in the genome database, which is estimated by a Nearest Sequenced Taxon Index (NSTI). Low 
values of NSTI, such as in our samples $(0.056 \pm 0.002 \mathrm{SE})$, illustrate a good coverage. We ran the same model than for species richness for the predicted number of functional genes involved in the 4 major pathway classes (KEGG-Level 1 classes, Supplementary Table 15, Supplementary Fig. 4). We used linear mixed models with lmer procedure in R version 3.1.1. We fitted a full model including climatic treatment as a continuous variable, plus sex and age classes ( 1 or $\geq 2$ years old) of individuals as covariates and every possible interaction. We further tested whether the predicted number of genes involved in the 22 KEGG-Level 2 pathway classes differed between climates by performing a PERMANOVA with $\mathrm{R}$ adonis function from the vegan package, with 999 permutations. Given the interaction between sex and climate on the 6 major functional groups, we performed this comparison between climates, sex and their interactions. We finally ran the analyses on these 22 KEGG-Level 2 classes within the 4 KEGG-Level 1 functional groups mentioned (with a p-value threshold of 0.0022 , Supplementary Table 16).

\section{Effect of climate change on populations' bacterial richness in the first experiment}

We calculated the impact of climate change on the richness at the population level, that is the total number of different OTUs per enclosure. We also calculated bacterial community variation at the host populations (i.e. enclosure) levels following ${ }^{20}$. We created OTUs presence-absence matrices for each enclosure. We then used the beta.div function by ${ }^{20}$ in $\mathrm{R}$ with 999 permutations to compute an estimate of total variance within each enclosure, using the Jaccard similarity index appropriate for presence-absence data. For these analyses, we excluded the 'intermediate' climate level as one of the enclosures in this level contained only 3 individuals in May 2013, and deriving conclusions at the population level from so few individuals could lead to spurious results. We thus compared the mean variance in bacterial community composition between 'warm' and 'present' climates with Wilcoxon tests.

\section{Pathways of impact of climate on bacterial richness in the first experiment}

We also investigated the mechanism by which climatic conditions affected bacterial richness (Supplementary Table 13). We modelled bacterial richness as an additive function of climatic conditions, individual condition (body mass), social context (lizard density), sexual context (number of sexual partners) and dietary context (number of insect and arachnid families in the enclosures), plus random enclosure effect with a linear mixed model, and derived all simpler models. We chose these variables as it has been shown that both individuals body condition, diet, sexual and social context could affect animals' microbiota ${ }^{21,6,22-24}$. Variables were previously centred and scaled ${ }^{16}$. Calculating Variance Inflation Factors with the corvif function from ${ }^{25}$ in $\mathrm{R}$ (http://www.highstat.com/Book2/HighstatLibV6.R) allowed to prove that multicollinearity was not an issue in our analysis (VIF $<3$, Supplementary Table $14,{ }^{16}$ ).

\section{Effect of short-term summer climate change on bacterial richness in the second experiment}

We additionally studied how climatic conditions affected extinction and colonization processes in our follow-up short-term summer experiment. For each individual, we calculated the rate of OTUs gone extinct during the summer (i.e. number of OTUs present in May but absent in September divided by the total number of OTUs present in May) and the rate of OTUs gained during the summer (i.e. number of OTUs present in September but not in May divided by the total number of OTUs present in September). We then applied the same mixed model method to investigate climatic impacts on proportions of new OTUs, extinction rates and total numbers of OTUs at the end of the summer (Tables S11-12)

\section{Effect of microbiota on host survival in the third experiment}


We investigated whether host future survival was related to bacterial richness in a common garden experiment using generalized linear mixed models with a logit link and a binomial distribution (Supplementary Table 17, Supplementary Fig. 5). The full model included lizard bacterial richness (centred and scaled) in interaction with climatic treatment the year before, plus age, sex and body condition (residuals of a linear model of body mass by body length, centred and scaled) as covariates, and random enclosure identity. Including age, sex and body condition allowed ensuring that impacts of bacterial richness did not depend on a possible link between bacterial richness and important individual characteristics that should play a role in survival. Multicollinearity was not an issue (Supplementary Table 18).

We finally investigated whether host future survival was related to the 49 bacterial OTUs with different relative abundance between present and warm climates selected using linear discrimant analyses (Supplementary Table 10). To do so, we first ran a principal component analyses on the relative abundance of the 49 OTUs using the FactoMineR package ${ }^{26}$. The first axis had an eigenvalue of 6.27 and differentiated the 49 OTUs differentially abundant in present and in warm climates (Supplementary Table 19). We therefore used this axis only to investigate survival generalized linear mixed models with a logit link and a binomial distribution. The full model included this axis (centred and scaled) in interaction with climatic treatment the year before, plus age, sex and body condition (residuals of a linear model of body mass by body length, centred and scaled) as covariates, and random enclosure identity. The averaged best model is shown in Supplementary Table 20. 


\section{Supplementary results}

\section{Effect of climate change on individual bacterial richness in the first experiment}

Climate change had a strong negative impact on total bacterial richness (Supplementary Table 1, Fig. 1a), and this effect was maintaining when removing unidentified OTUs (Supplementary Table 2). The effect did not rely on issues of sample sequencing depth as there were no significant differences between climates in sample sequencing depth and coverage (Supplementary Fig. 1a-d), and removing samples with low sample sequencing depth did not change the results (Supplementary Table 3). Further, the effect of climatic conditions on bacterial richness did not rely only on a disappearance of very rare bacterial OTUs as truncating the data with several abundance/prevalence cut-offs yielded the same effect (Supplementary Table 4). This suggests that our effect did not result from the disappearance of rare bacterial OTUs only. The accumulation curves (i.e. the number of OTUs with increasing number of lizard individuals sampled, Supplementary Fig. 3) further showed that the lower number of OTUs in warm climatic treatments resulted from both a decrease of the number of bacterial taxa at the individual level and a decrease in the number of host individuals due to increased mortality ${ }^{2}$.

Looking on the impact of climate on the most diverse phyla (5 phyla with the highest number of OTUs plus unidentified OTUs), we found a significant effect of climatic treatment (Supplementary Tables 5 and 6), without interactions with phylum identity. Separate models by phylum showed a significant impact of climatic conditions for Bacteroidetes, Proteobacteria, Firmicutes and Unidentified taxa, but not on Fusobacteria and Actinobacteria (Supplementary Table 7).

\section{Effect of climate change on individual community structure in the first experiment}

Climate change had no impact on bacterial community evenness (Supplementary Table 8), and no effect on dissimilarities between hosts' microbial communities visualized through a Non Metric Dimensional Scaling analysis (Fig. 1c).

We then further explored what bacterial taxa differed between the climatic treatments. The relative abundance in major bacterial groups differed between climates (Supplementary Table 9). The relative abundance was higher in present than in warm climates for Bacteroidetes and Firmicutes. In contrast, the relative abundance of Proteobacteria tended to be higher in warmer climates (Supplementary Table 9, Fig. 1d).

We further explored the bacterial OTUs responsible for differences in diversity and community structure between present and warm climates (Supplementary Table 10) and identified 24 OTUs whose relative abundance was significantly lower in warmer climate (Supplementary Table 10) according to our quite conservative approach [consensus between linear discriminant analysis (LDA score > 2) and Mann-Whitney test ( $<$ < 0.005)]. These are mainly members Bacteroidales and Lachnospiraceae, two groups that are typical from the gut environment in reptiles ${ }^{27,28}$. Reduction of these potential important members of the gut microbiota may hence be deleterious for the host through e.g. a reduced nutrient assimilation or facilitate the colonization of the gut by pathogens. Accordingly, some OTUs had a higher relative abundance in warmer climates (Supplementary Table 10) and were affiliated to Gammaproteobacteria and Actinobacteria, of which certain members are notorious pathogens. Although further analyses should be required to confirm this hypothesis, it supports previous findings in a laboratory warming experiments on tadpoles ${ }^{29}$. 
We further studied the differences of bacterial functional profiles between warm and present climates (Tables S15-S16). We found that functional features associated to metabolism, information processing and cellular processes were differentially abundant between climates, but in different directions for females and males (Tables S15-S16). For example, metabolismassociated functional features (e.g. lipid and energy metabolism) increased with warming in male hosts, and conversely decreased in female hosts. This difference in functional profiles was also supported by a PERMANOVA showing a significant interaction between climates and $\operatorname{sex}\left(\mathrm{F}_{1,146}=9.35, \mathrm{p}=0.001\right)$. The functional profiles were impacted by climatic conditions in males $\left(\mathrm{F}_{1,76}=4.37, \mathrm{p}=0.018\right)$ and females $\left(\mathrm{F}_{1,70}=5.06, \mathrm{p}=0.004\right)$, but in different ways (Supplementary Fig. 4, Supplementary Table 15).

\section{Effect of climate change on populations' bacterial richness in the first experiment}

At the population level, we observed a $34 \%$ decrease of total bacterial diversity with warming (warm climate: $2451 \pm 1194$ SE, present climate: $3708 \pm 938$ SE). Warming also caused greater variability in microbial community composition within host populations (mean Jaccard index within a population; present climate: $0.446 \pm 0.002$, warm climate: $0.458 \pm$ $\left.0.004, \chi^{2}=3.86, \mathrm{p}=0.0495\right)$.

\section{Pathways of impact of climate on bacterial richness in the first experiment}

Investigating the mechanism by which climatic conditions affected bacterial richness (Supplementary Table 13), we found that the averaged best model included climatic treatment, lizard density and both number of arachnid and insect families. However only the climate effect had a significant negative impact on bacterial richness while lizard density tended to be positively related to richness (Supplementary Table 13).

\section{Effect of short-term summer climate change on bacterial richness in the second experiment}

We additionally studied how climatic conditions affected extinction and colonization processes in our follow-up short-term summer experiment. Bacterial richness increased over the summer in both present and warm climates (Supplementary Table 11). This could be potentially due to a more diversified and/or different diet in the summer (seasonal shifts or increases in invertebrate communities), to variations in social and sexual interactions throughout the year, to differences in lizards' physiology throughout the year (e.g. post laying phase for the females in July, or preparation for hibernation in September for all lizards) or to a seasonal difference in temperature, although our data does not allow to infer the precise reason for this seasonal change. However, climate warming led to higher bacterial extinction rates, while there were no differences in the proportion of new OTUs (Supplementary Table 12). Hence, the increase of bacterial richness over the summer was stronger in present climate (climate effect: estimate $\pm \mathrm{SE}:-130 \pm 46, \mathrm{t}=-2.8, \mathrm{p}=0.004$, mean change in OTUs number over the summer: $+134 \pm 194$ and $+4 \pm 131$ in present and warm climates respectively) and resulted in differences in bacterial richness after climatic treatments (climate effect: estimate \pm SE: $-128 \pm 56, \mathrm{p}=0.026, \mathrm{RI}=0.63$, Supplementary Table 11 , mean OTUs number in September: $526 \pm 168$ and $400 \pm 128$ in present and warm climates respectively) while there were no differences between treatments at the beginning of the experiment $\left(\mathrm{F}_{1,82}=0.97, \mathrm{p}=\right.$ $0.33)$.

\section{Effect of microbiota on host survival in the third experiment}

Investigating whether host future survival was related to bacterial richness in a common garden experiment, the averaged best model included bacterial richness, climatic treatment the 
year before, body condition and sex, however only bacterial richness and climatic treatment were significant (Supplementary Table 17, Supplementary Fig. 5). A further analysis showed that survival was positively related to a principal component summarising the 49 bacterial OTUs with different relative abundance between present and warm climates described in Supplementary Table 10 (Supplementary Table 20).

\section{References}

1. Legrand, D. et al. Nature Methods 9, 828-833 (2012).

2. Bestion, E., Teyssier, A., Richard, M., Clobert, J. \& Cote, J. PLoS Biol 13, e1002281 (2015).

3. Bestion, E., Clobert, J. \& Cote, J. Ecol Lett 18, 1226-1233 (2015).

4. IPCC (Cambridge University Press: Cambridge, United Kingdom and New York, NY, USA, 2013).

5. Bestion, E., Cucherousset, J., Teyssier, A. \& Cote, J. Oikos 124, 1597-1603 (2015).

6. White, J., Richard, M., Massot, M. \& Meylan, S. PLoS ONE 6, e22339 (2011).

7. Colston, T.J., Noonan, B.P. \& Jackson, C.R. PLOS ONE 10, e0128793 (2015).

8. Kohl, K.D. et al. Mol Ecol n/a-n/a (2016).doi:10.1111/mec.13921

9. Teyssier, A., Bestion, E., Richard, M. \& Cote, J. Behavioral Ecology 25, 723-733 (2014).

10. Fliegerova, K. et al. Anaerobe 29, 80-84 (2014).

11. Taberlet, P. et al. Molecular ecology 21, 1816-20 (2012).

12. Boyer, F. et al. Mol Ecol Resour 16, 176-182 (2015).

13. Wang, Q., Garrity, G.M., Tiedje, J.M. \& Cole, J.R. Appl Environ Microbiol 73, 52615267 (2007).

14. Bates, D., Maechel, M. \& Bolker, B. (2014).at <http://CRAN.Rproject.org/package $=$ lme4>

15. R Core Team (R Foundation for Statistical Computing: Vienna, Austria, 2014).at $<$ http://www.R-project.org/>

16. Grueber, C.E., Nakagawa, S., Laws, R.J. \& Jamieson, I.G. Journal of Evolutionary Biology 24, 699-711 (2011).

17. Barton, K.A. (2012).at <http://CRAN.R-project.org/package=MuMIn>

18. Langille, M.G.I. et al. Nat Biotech 31, 814-821 (2013).

19. Kanehisa, M. \& Goto, S. Nucl Acids Res 28, 27-30 (2000).

20. Legendre, P. \& De Cáceres, M. Ecology Letters 16, 951-963 (2013).

21. Lurgi, M., López, B.C. \& Montoya, J.M. Phil. Trans. R. Soc. B 367, 3050-3057 (2012).

22. Bolnick, D.I. et al. Ecology Letters 17, 979-987 (2014).

23. Carey, H.V., Walters, W.A. \& Knight, R. American Journal of Physiology - Regulatory, Integrative and Comparative Physiology 304, R33-R42 (2013).

24. Dillon, M.E., Wang, G. \& Huey, R.B. Nature 467, 704-706 (2010).

25. Zuur, A.F., Ieno, E.N., Walker, N.J., Saveliev, A.A. \& Smith, G.M. (New York, 2009).

26. Lê, S., Josse, J., Husson, F. \& others Journal of statistical software 25, 1-18 (2008).

27. Waite, D.W. \& Taylor, M. Front. Microbiol. 6, (2015).

28. Hong, P.-Y., Wheeler, E., Cann, I.K.O. \& Mackie, R.I. ISME J 5, 1461-1470 (2011).

29. Kohl, K.D. \& Yahn, J. Environ Microbiol 18, 1561-1565 (2016). 


\section{Supplementary Figures}
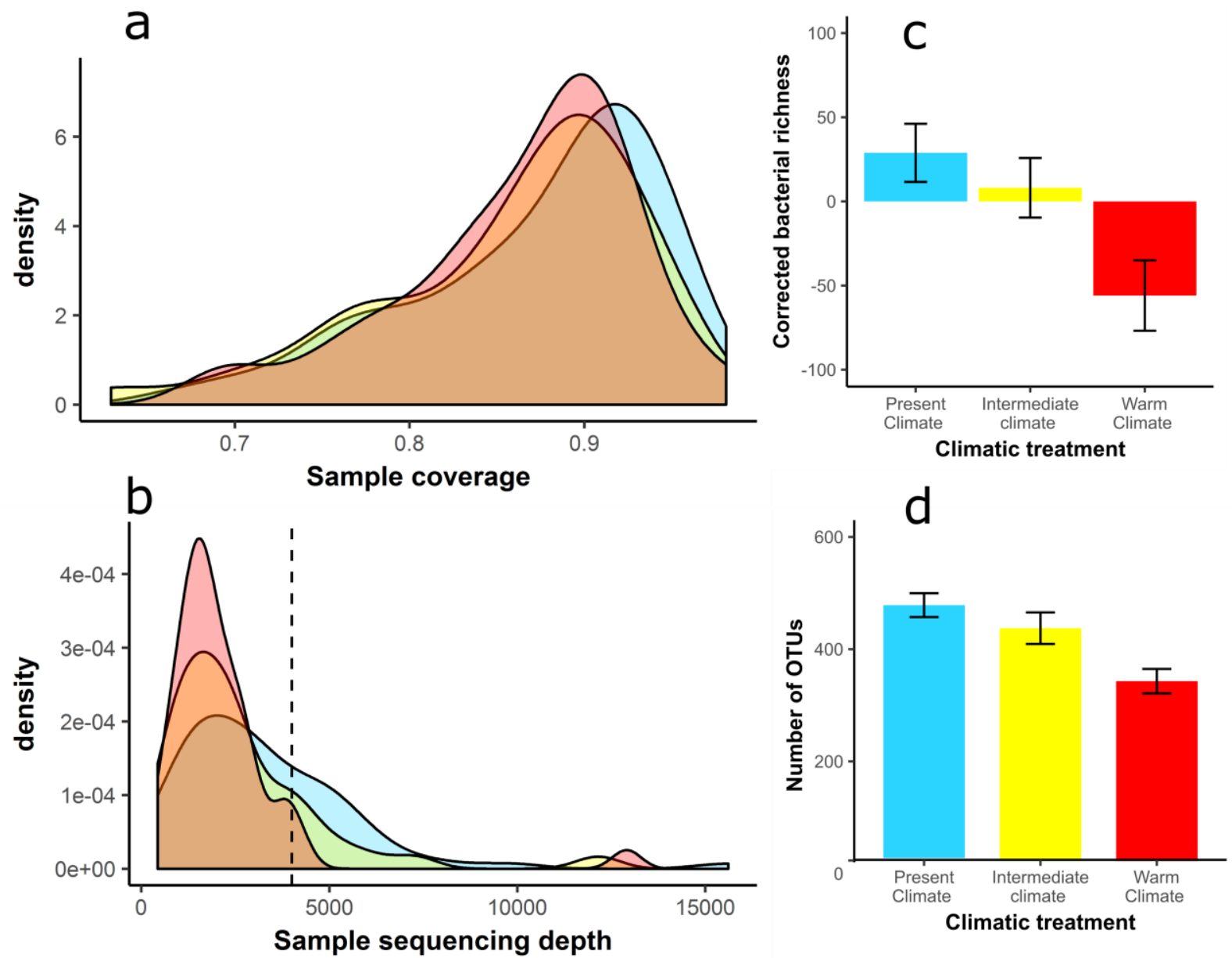

Figure 1: a: Sample sequencing coverage (calculated as 1-(singletons/total number of sequences)) as a function of climatic treatment. There is no significant difference between treatments in sample sequencing coverage (mixed model with coverage as a function of climate treatments plus nested random intercept enclosure, $\chi^{2}=1.08, \mathrm{df}=2, \mathrm{p}=0.58$ ). $\mathbf{b}$ : Sample number of sequences as a function of climatic treatment (blue: present climate, yellow: intermediate climate, red: warm climate). There is no significant difference between treatments in the number of sequences (mixed model with $\log$ (number of sequences) as a function of climate treatments plus nested random intercept enclosure, $\chi^{2}=4.91, \mathrm{df}=2, \mathrm{p}=$ 0.09). c: Impact of climatic conditions on individual bacterial richness corrected for the number of sequences (mean \pm SEM). We analysed the effect of climatic conditions on the residuals from a model "bacterial richness $\sim$ number of sequences". Climatic conditions have an impact on corrected bacterial richness (climate as a covariate, estimate \pm SE $=-7.279 \pm$ $\left.3.556, \chi^{2}=4.19, \mathrm{df}=1, \mathrm{p}=0.04\right)$. $\mathrm{d}$ : Impact of climatic conditions on individual bacterial richness for individuals with less than 4000 sequences (mean number of taxa \pm SEM). In this sub-sample, the number of sequences are equal among treatments $(\chi 2=1.49, \mathrm{df}=2, \mathrm{p}=0.47)$ and climatic conditions have an impact on corrected bacterial richness (climate as a covariate in a model including climatic treatment, number of sequences and random enclosure, estimate $\left.\pm \mathrm{SE}=-6.18 \pm 2.26, \chi^{2}=7.48, \mathrm{p}=0.006\right)$. 


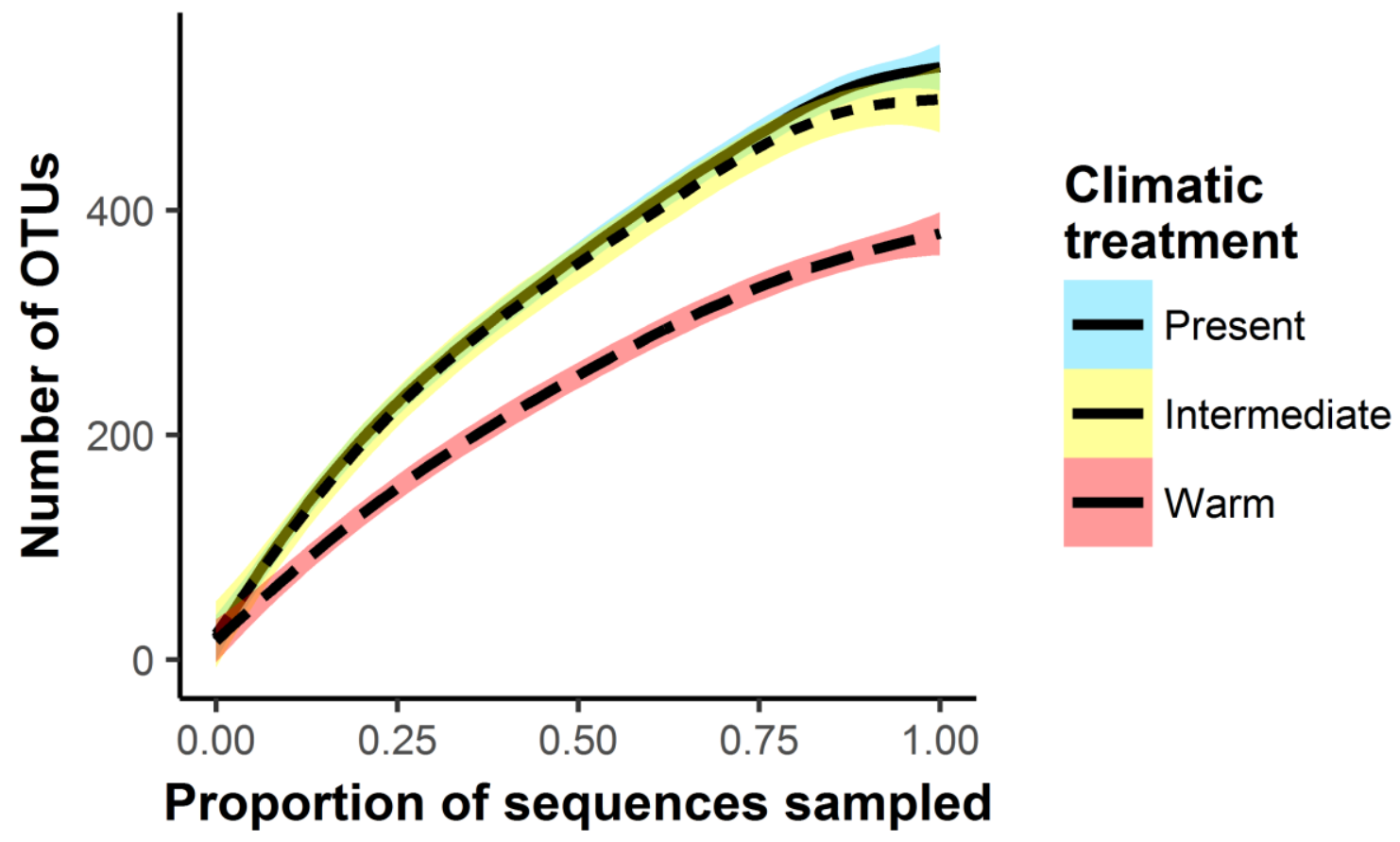

Figure 2: Rarefaction curves of the number of OTUs with increasing number of sequences sampled broken down by treatment. 


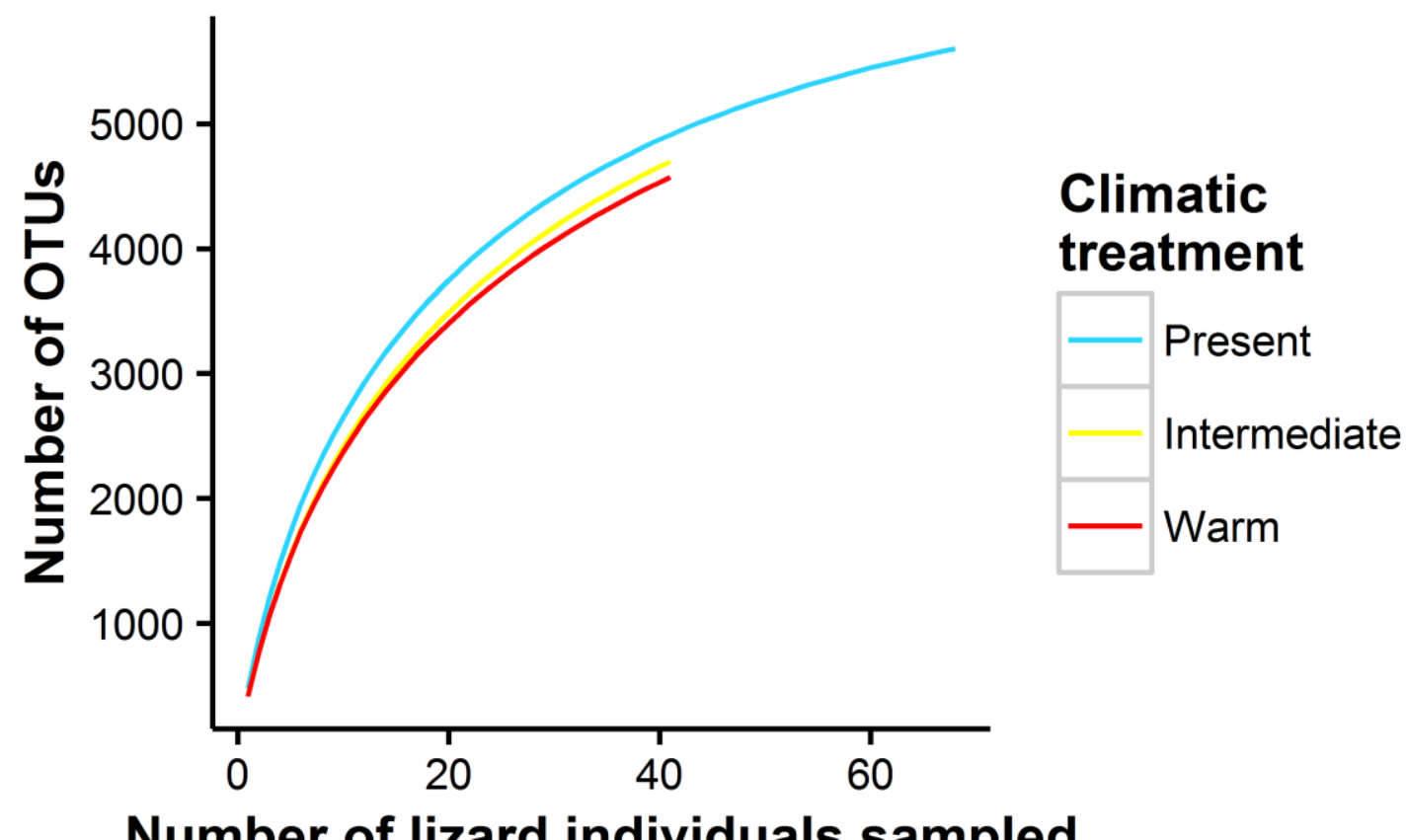

Number of lizard individuals sampled

Figure 3: Accumulation curves of the number of OTUs with increasing number of lizard individuals sampled broken down by treatment. The lower number of OTUs in warm climatic treatments is due to both a decrease of the number of bacterial taxa at the individual level and to a decrease in the number of host individuals due to increased mortality. 
Figure 4: Impact of climatic conditions on the predicted number of genes involved in main functions groups [Metabolism (a), Environmental (b) and Genetic (c) Information Processing] of bacteria selected using Picrust (see Supplementary Table 15, mean \pm SEM).

a)

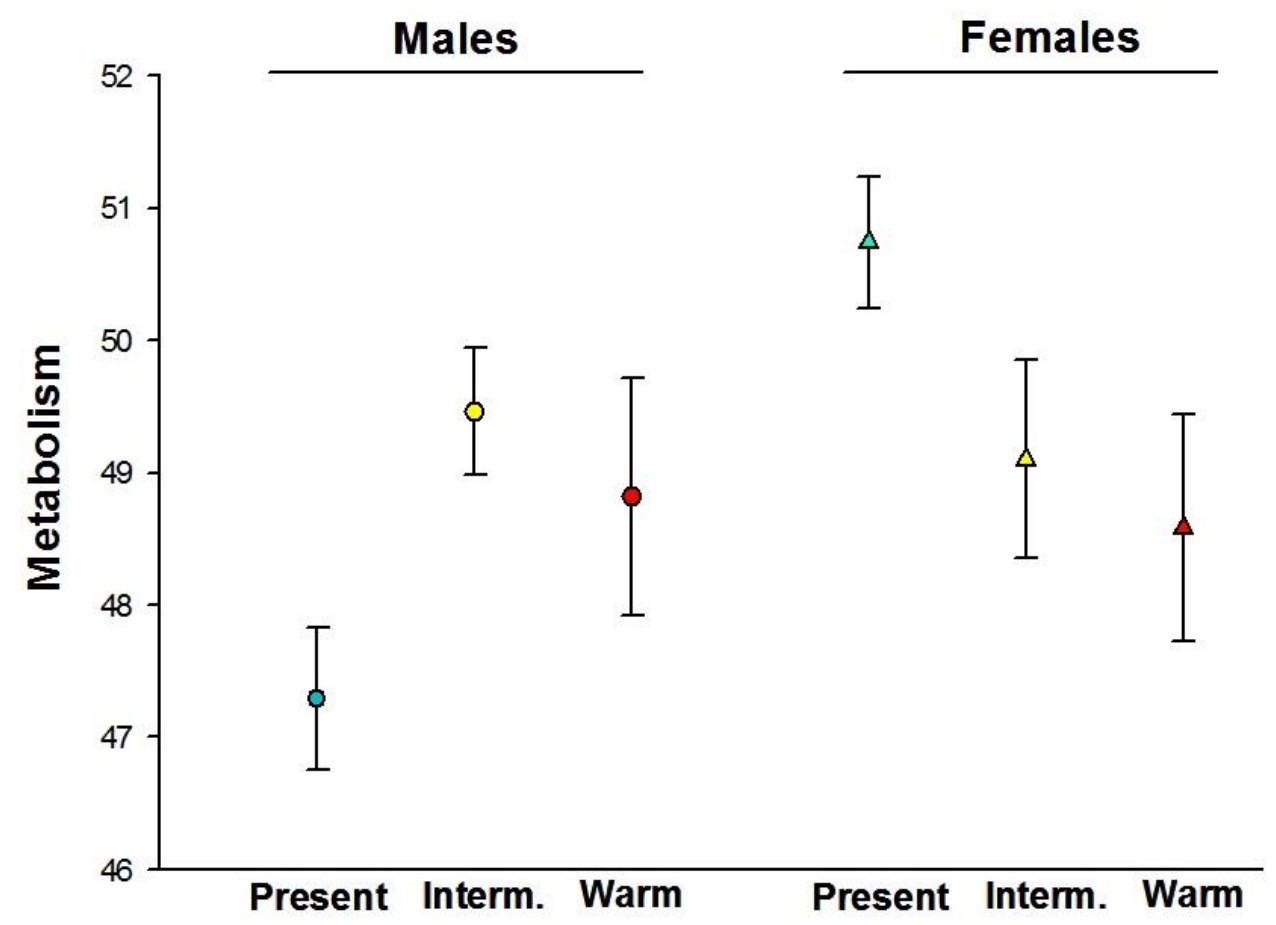

Climatic Treatments

b)

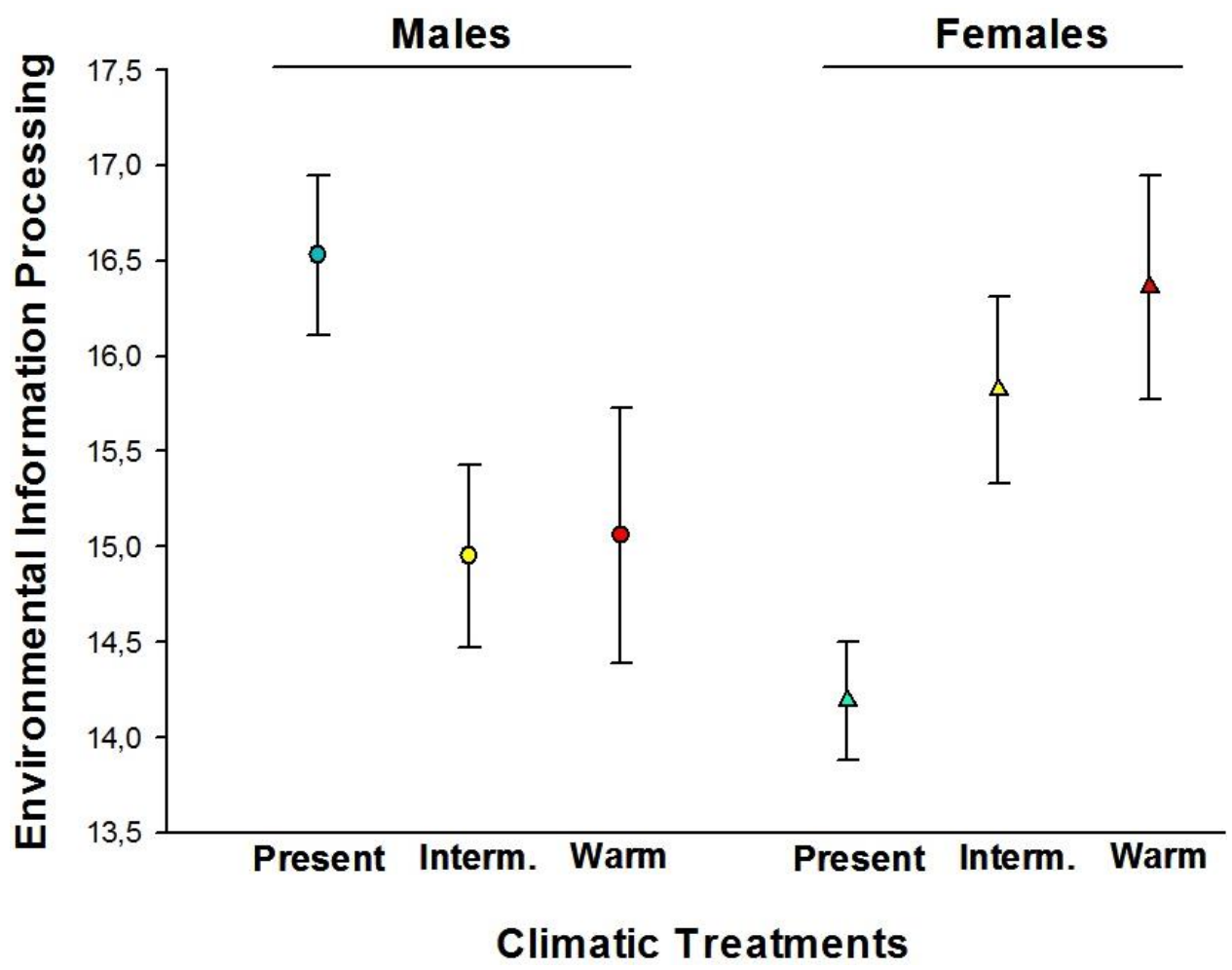


c)

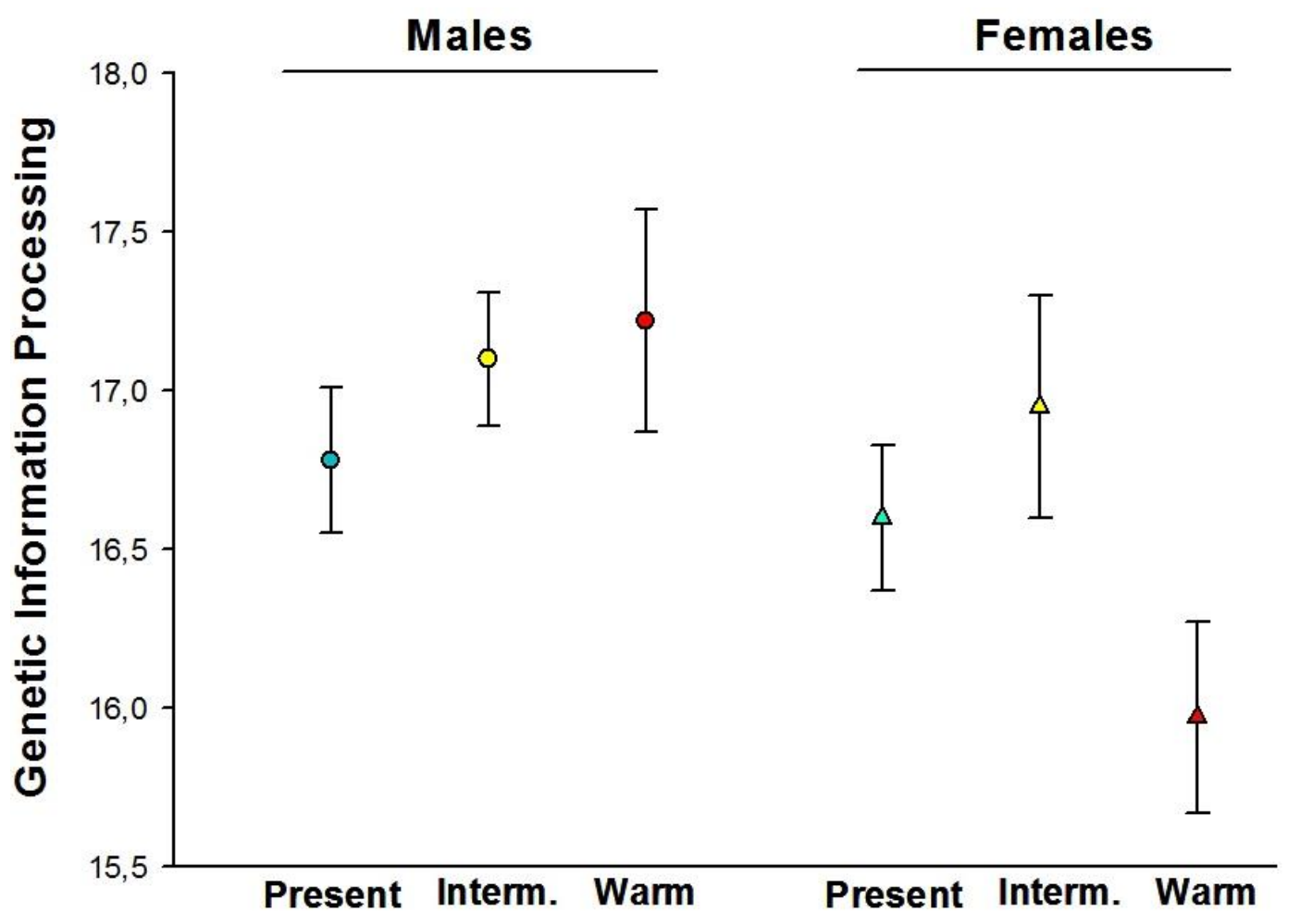

Climatic Treatments 


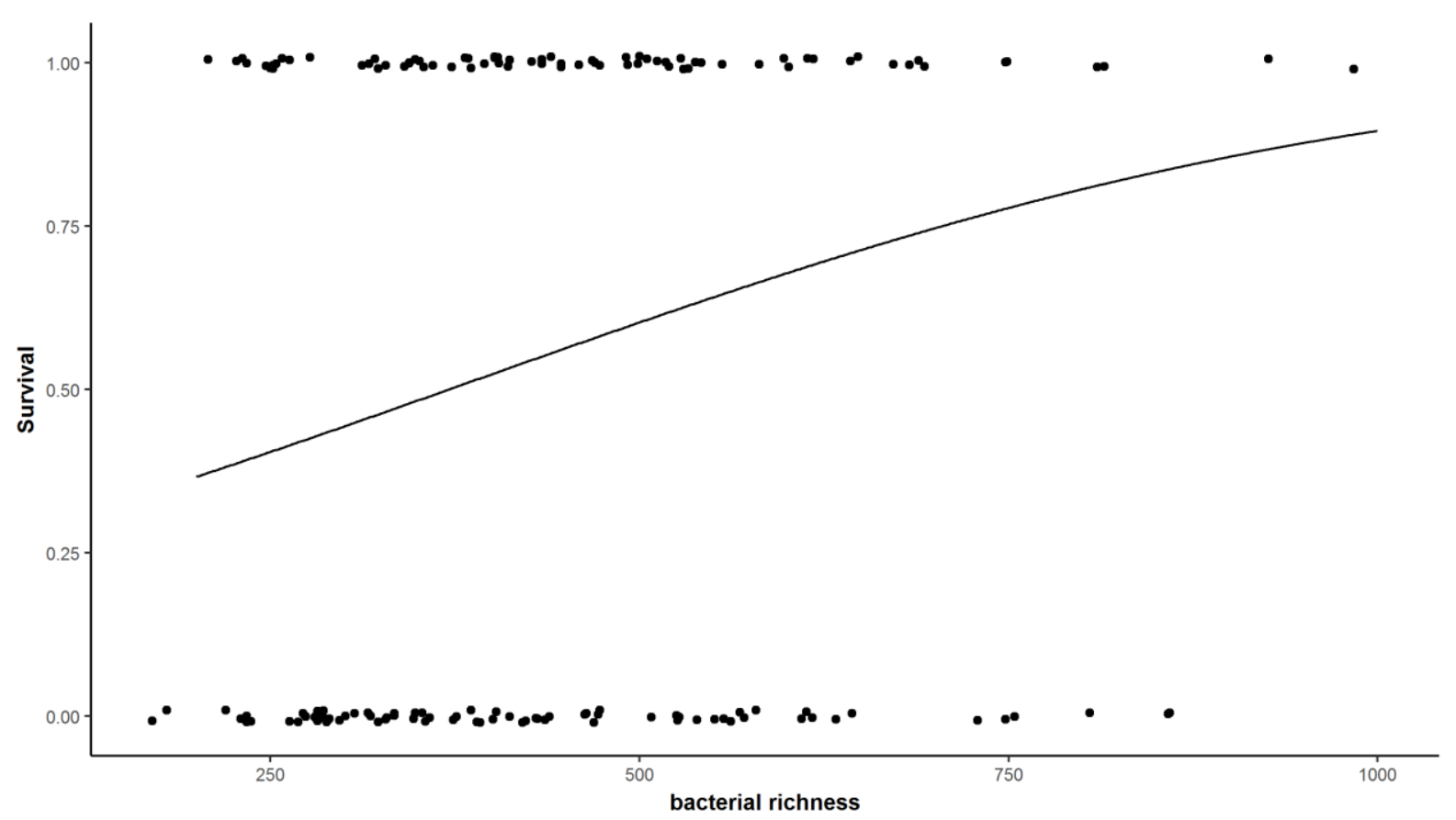

Figure 5: Survival as a function of bacterial richness. Plot of the predicted values from the averaged best model investigating survival as a function of bacterial richness, sex, body condition and random enclosure identity (see Supplementary Table 17). 


\section{Supplementary Tables}

Table 1: Impact of climate, lizard age, sex and sample sequencing depth on bacterial richness (total OTUs number). $\mathrm{N}=150$ lizards. Symbols denote different levels of significance of the $\mathrm{p}-$ values: $* * * \mathrm{p}<0.001, * * \mathrm{p}<0.01, * \mathrm{p}<0.05, . \mathrm{p}<0.10$.

\begin{tabular}{lllll}
\hline Parameter & Estimate & SE & p-value & $\begin{array}{l}\text { Relative } \\
\text { Importance }\end{array}$ \\
\hline Climate & -7.1 & 3.3 & $0.017^{*}$ & 1.00 \\
Sample & & & & \\
sequencing depth & 83.3 & 10.9 & $>0.001^{* * *}$ & 1.00 \\
Sex & 18.9 & 21.3 & 0.38 & 0.33 \\
\hline
\end{tabular}


Table 2: Impact of climatic conditions, lizard age and sex-classes on individual gut bacteria richness including only identified OTUS. Models were derived from a global model including climatic treatment, age and sex and every interactions plus sample sequencing depth (centred and scaled) and its interaction with climatic condition as a covariate and enclosure identity as a random intercept. The top best models $(\triangle \mathrm{AICc}<2)$ were then used in a model averaging method following Grueber et al (2011). Symbols denote different levels of significance of the p-values: $* * * \mathrm{p}<0.001, * * \mathrm{p}<0.01, * \mathrm{p}<0.05, \mathrm{p}<0.10$

\begin{tabular}{lllll}
\hline Parameter & Estimate & SE & p-value & $\begin{array}{l}\text { Relative } \\
\text { Importance }\end{array}$ \\
\hline Climate & -6.18 & 2.32 & $0.008 * *$ & 1.00 \\
Age & 8.43 & 15.03 & 0.58 & 0.30 \\
Sex & 15.57 & 14.30 & 0.28 & 0.20 \\
Sample & & & & \\
sequencing depth & 52.78 & 7.36 & $>0.001 * * *$ & 1.00 \\
\hline
\end{tabular}


Table 3: Impact of climatic conditions, lizard age and sex-classes on individual gut bacteria richness (total OTUs number) including only samples for which the sample sequencing depth was $>1000$ reads $(\mathrm{N}=132$ lizards $)$. Models were derived from a global model including climatic treatment, age and sex and every interactions plus sample sequencing depth (centred and scaled) and its interaction with climatic condition as a covariate and enclosure identity as a random intercept. The top best models $(\triangle \mathrm{AICc}<2)$ were then used in a model averaging method following Grueber et al (2011). Symbols denote different levels of significance of the p-values: $* * * \mathrm{p}<0.001, * * \mathrm{p}<0.01, * \mathrm{p}<0.05, \mathrm{p}<0.10$.

\begin{tabular}{lllll}
\hline Parameter & Estimate & SE & p-value & $\begin{array}{l}\text { Relative } \\
\text { Importance }\end{array}$ \\
\hline Climate & -8.6 & 3.4 & $0.011^{*}$ & 1.00 \\
$\begin{array}{l}\text { Sex } \\
\text { Sample }\end{array}$ & 28.1 & 23.7 & 0.24 & 0.40 \\
sequencing depth & 73.8 & 12.1 & $>0.001^{* * * *}$ & 1.00 \\
\hline
\end{tabular}


Table 4: Impact of climatic conditions on individual gut bacteria richness when keeping OTUs with different minimum abundance and prevalence cut-offs .:10p, 20p: including OTUs present in at least $10 \%$ (resp. $20 \%$ ) of the samples. 20a, 50a: including only the $20 \%$ (resp. 50 $\%$ ) more abundant OTUs. 10p_20a, 10p_50a, 20p_20a, 20p_50a: including only the 20\% (or $50 \%$ ) more abundant OTUs and present in at least than $10 \%$ (or $20 \%$ ) of the samples. Models included bacterial richness as a function of climatic treatment plus enclosure identity as a random intercept. Estimate and Standard Error of the climatic treatment are given, as well as results from a Type II Wald chisquare test investigating the significance of the effect of climatic treatment. Symbols denote different levels of significance of the $\mathrm{p}$-values: $* * * \mathrm{p}<$ $0.001, * * \mathrm{p}<0.01, * \mathrm{p}<0.05, \mathrm{p}<0.10$.

\begin{tabular}{|c|c|c|c|c|}
\hline Cut-off & Estimate & $\mathrm{SE}$ & $\begin{array}{l}\text { Wald } \\
\text { chisquare } \\
(\mathrm{df}=1)\end{array}$ & p-value \\
\hline $10 p$ & -6.47 & 2.65 & 5.95 & $0.015 *$ \\
\hline $20 p$ & -3.41 & 1.64 & 4.34 & $0.037 *$ \\
\hline $20 \mathrm{a}$ & -11.03 & 4.15 & 7.06 & $0.008 * *$ \\
\hline $50 \mathrm{a}$ & -6.95 & 2.98 & 5.43 & $0.020 *$ \\
\hline 10p_20a & -5.01 & 2.23 & 5.07 & $0.024 *$ \\
\hline 10p_50a & -2.62 & 1.43 & 3.34 & 0.068 . \\
\hline 20p_20a & -2.62 & 1.37 & 3.65 & 0.056 . \\
\hline 20p_50a & -1.19 & 0.86 & 1.95 & 0.163 \\
\hline
\end{tabular}


Table 5: Impact of climatic conditions on bacterial richness within each of the most diverse phyla. Models were derived from a global repeated mixed model including climatic treatment, phylum identity and their interaction plus enclosure identity and individual identity as random intercepts. The top best models $(\triangle \mathrm{AICc}<2)$ were then used in a model averaging method following Grueber et al (2011). For a list of all models with $\triangle$ AICc see Supplementary Table 6. Symbols denote different levels of significance of the p-values: $* * * p<0.001, * * \mathrm{p}<0.01$, $* \mathrm{p}<0.05, \mathrm{p}<0.10$.

\begin{tabular}{lllll}
\hline Parameter & Estimate & SE & p-value & $\begin{array}{l}\text { Relative } \\
\text { Importance }\end{array}$ \\
\hline Climate & -0.04 & 0.02 & $0.025^{*}$ & 0.73 \\
\hline
\end{tabular}


Table 6: Comparison of the models investigating bacterial richness within each of the most diverse phyla as a function of climatic treatment and phylum identity through their AICc. Models were derived from a global repeated mixed model including climatic treatment, phylum identity and their interaction plus enclosure identity and individual identity as random intercepts. The top best models $(\triangle \mathrm{AICc}<2)$ were then used in a model averaging method following Grueber et al (2011), see Supplementary Table 5.

\begin{tabular}{lccc}
\hline Model & df & AICc & $\Delta$ AICc \\
\hline Temperature treatment $+(1 \mid$ Enclosure $)+(1 \mid$ Individual $)$ & 5 & 2392.6 & 0.00 \\
$(1 \mid$ Enclosure $)+(1 \mid$ Individual $)$ & 4 & 2394.6 & 1.97 \\
$\begin{array}{l}\text { Temperature treatment * Phylum identity }+(1 \mid \text { Enclosure })+ \\
(1 \mid \text { Individual })\end{array}$ & 15 & 2399.6 & 6.97 \\
$\begin{array}{l}\text { Temperature treatment }+ \text { Phylum identity }+(1 \mid \text { Enclosure })+ \\
(1 \mid \text { Individual })\end{array}$ & 10 & 2402.8 & 10.18 \\
Phylum identity $+(1 \mid$ Enclosure $)+(1 \mid$ Individual $)$ & 9 & 2404.8 & 12.13 \\
\hline
\end{tabular}


Table 7: Impact of climatic treatment for each of the most diverse phyla investigated through separate spearman tests. P-values of the spearman test of $\mathrm{nb}$ taxa as a function of climate, first raw and then corrected through various corrections for multiple testing using HolmsBonferroni and Benjamini and Hochberg corrections. Symbols denote different levels of significance of the $\mathrm{p}$-values: $* * * \mathrm{p}<0.001, * * \mathrm{p}<0.01, * \mathrm{p}<0.05, . \mathrm{p}<0.10$.

\begin{tabular}{lccc}
\hline & Raw p-value & Holms-Bonferroni & Benjamini and Hochberg \\
\hline Proteobacteria & $0.015^{*}$ & $0.043^{*}$ & $0.022^{*}$ \\
Firmicutes & $>0.001^{* * *}$ & $>0.001^{* * *}$ & $>0.001^{* * *}$ \\
Bacteroidetes & $>0.001^{* * *}$ & $0.001^{* *}$ & $>0.001^{* * *}$ \\
Actinobacteria & 0.326 & 0.329 & 0.329 \\
Fusobacteria & 0.110 & 0.219 & 0.131 \\
Unidentified & $0.001 * *$ & $0.004^{* *}$ & $0.002 * *$ \\
\hline
\end{tabular}


Table 8: Impact of climatic conditions, lizard age and sex-classes on individual gut bacteria evenness. Models were derived from a global model including climatic treatment, age and sex and every interactions plus enclosure identity as a random intercept. The top best models $(\triangle \mathrm{AICc}<2)$ were then used in a model averaging method following Grueber et al $(2011)$. Symbols denote different levels of significance of the p-values: $* * * \mathrm{p}<0.001, * * \mathrm{p}<0.01, *$ $\mathrm{p}<0.05, . \mathrm{p}<0.10$.

\begin{tabular}{lllll}
\hline Parameter & Estimate & SE & p-value & $\begin{array}{l}\text { Relative } \\
\text { Importance }\end{array}$ \\
\hline Age & -0.03 & 0.03 & 0.22 & 0.29 \\
Sex & 0.02 & 0.03 & 0.52 & 0.17 \\
Climate & -0.001 & 0.003 & 0.69 & 0.15 \\
\hline
\end{tabular}


Table 9: Difference in relative abundances in most diverse bacterial groups between present, intermediate and warm climates. Symbols denote different levels of significance of the $\mathrm{p}$ values: $* * * \mathrm{p}<0.001, * * \mathrm{p}<0.01, * \mathrm{p}<0.05, . \mathrm{p}<0.10$.

\begin{tabular}{ccc}
\hline Phylum & Chi-square & p-value \\
\hline Proteobacteria & 5.781 & 0.056. \\
Firmicutes & 8.908 & $0.012 *$ \\
Bacteroidetes & 7.623 & $0.022 *$ \\
Actinobacteria & 4.339 & 0.114 \\
Fusobacteria & 1.434 & 0.488 \\
Unidentified & 1.695 & 0.429 \\
\hline
\end{tabular}


Table 10a: Bacterial OTUs with different relative abundances between climatic treatments. These OTUs were selected using linear discriminant scores (LDA > 2.0) and Mann-Whitney test ( OTUs for which we could not assign a taxon that had a relative abundance higher in the present or warm climate respectively $($ LDA $=2.12-3.14$, p_MW $<$ $0.0035)$.

\begin{tabular}{|c|c|c|c|c|c|c|c|}
\hline Phylum & Class & Order & Family & Genus & Highest for: & LDA & p_MW \\
\hline Actinobacteria & Actinobacteria & Actinomycetales & Nakamurellaceae & Nakamurella & Present Clim. & 2.43 & 0.0021 \\
\hline Bacteroidetes & Bacteroidia & Bacteroidales & NA & NA & Present Clim. & 2.35 & 0.0042 \\
\hline Bacteroidetes & Bacteroidia & Bacteroidales & NA & NA & Present Clim. & 2.60 & 0.0016 \\
\hline Bacteroidetes & Bacteroidia & Bacteroidales & Bacteroidaceae & Bacteroides & Present Clim. & 2.07 & 0.0050 \\
\hline Bacteroidetes & Bacteroidia & Bacteroidales & NA & NA & Present Clim. & 2.25 & 0.0009 \\
\hline Bacteroidetes & Bacteroidia & Bacteroidales & Porphyromonadaceae & NA & Present Clim. & 3.22 & 0.0019 \\
\hline Firmicutes & Clostridia & Clostridiales & Lachnospiraceae & NA & Present Clim. & 2.42 & 0.0032 \\
\hline Firmicutes & Clostridia & Clostridiales & Lachnospiraceae & NA & Present Clim. & 2.50 & 0.0006 \\
\hline Firmicutes & Clostridia & Clostridiales & Lachnospiraceae & NA & Present Clim. & 2.38 & 0.0013 \\
\hline Firmicutes & Clostridia & Clostridiales & Lachnospiraceae & NA & Present Clim. & 2.54 & 0.0045 \\
\hline Firmicutes & Clostridia & Clostridiales & Lachnospiraceae & NA & Present Clim. & 2.31 & 0.0029 \\
\hline Firmicutes & Clostridia & Clostridiales & Lachnospiraceae & NA & Present Clim. & 2.21 & 0.0017 \\
\hline Firmicutes & Clostridia & Clostridiales & Lachnospiraceae & NA & Present Clim. & 3.38 & 0.0031 \\
\hline Firmicutes & Clostridia & Clostridiales & NA & NA & Present Clim. & 2.32 & 0.0042 \\
\hline Firmicutes & Clostridia & Clostridiales & NA & NA & Present Clim. & 2.31 & 0.0008 \\
\hline Firmicutes & Clostridia & Clostridiales & Lachnospiraceae & Clostridium & Present Clim. & 2.67 & 0.0041 \\
\hline Firmicutes & NA & NA & NA & NA & Present Clim. & 2.28 & 0.0047 \\
\hline Firmicutes & Clostridia & Clostridiales & Lachnospiraceae & NA & Present Clim. & 2.16 & 0.0019 \\
\hline Firmicutes & NA & NA & NA & NA & Present Clim. & 2.93 & 0.0008 \\
\hline Firmicutes & NA & NA & NA & NA & Present Clim. & 2.61 & 0.0000 \\
\hline Firmicutes & Clostridia & Clostridiales & NA & NA & Present Clim. & 2.38 & 0.0047 \\
\hline Firmicutes & Clostridia & Clostridiales & Lachnospiraceae & NA & Present Clim. & 2.48 & 0.0036 \\
\hline Proteobacteria & Deltaproteobacteria & Desulfovibrionales & Desulfovibrionaceae & NA & Present Clim. & 2.38 & 0.0016 \\
\hline Proteobacteria & NA & NA & NA & NA & Present Clim. & 2.98 & 0.0014 \\
\hline
\end{tabular}


Table 10b: Bacterial OTUs with different relative abundances between climatic treatments.

\begin{tabular}{|c|c|c|c|c|c|c|c|}
\hline Phylum & Class & Order & Family & Genus & Highest for: & LDA & P_MW \\
\hline Actinobacteria & Actinobacteria & Actinomycetales & NA & NA & Warm Clim. & 2.97 & 0.0001 \\
\hline Proteobacteria & NA & NA & NA & NA & Warm Clim. & 2.64 & 0.0038 \\
\hline Proteobacteria & Gammaproteobacteria & NA & NA & NA & Warm Clim. & 2.14 & 0.0027 \\
\hline Proteobacteria & NA & NA & NA & NA & Warm Clim. & 3.21 & 0.0011 \\
\hline Proteobacteria & NA & NA & NA & NA & Warm Clim. & 3.06 & 0.0027 \\
\hline Proteobacteria & NA & NA & NA & NA & Warm Clim. & 2.99 & 0.0005 \\
\hline Proteobacteria & NA & NA & NA & NA & Warm Clim. & 2.24 & 0.0011 \\
\hline Proteobacteria & Gammaproteobacteria & NA & NA & NA & Warm Clim. & 2.31 & 0.0039 \\
\hline Unidentified & NA & NA & NA & NA & Present Clim. & 2,22 & 0,0012 \\
\hline Unidentified & NA & NA & NA & NA & Present Clim. & 2,28 & 0,0004 \\
\hline Unidentified & NA & NA & NA & NA & Present Clim. & 2,89 & 0,0015 \\
\hline Unidentified & NA & NA & NA & NA & Present Clim. & 2,37 & 0,0006 \\
\hline Unidentified & NA & NA & NA & NA & Present Clim. & 2,53 & 0,0035 \\
\hline Unidentified & NA & NA & NA & NA & Present Clim. & 2,12 & 0,0013 \\
\hline Unidentified & NA & NA & NA & NA & Present Clim. & 2,39 & 0,0028 \\
\hline Unidentified & NA & NA & NA & NA & Present Clim. & 2,69 & 0,0022 \\
\hline Unidentified & NA & NA & NA & NA & Present Clim. & 2,12 & 0,0026 \\
\hline Unidentified & NA & NA & NA & NA & Present Clim. & 2,49 & 0,0024 \\
\hline Unidentified & NA & NA & NA & NA & Present Clim. & 2,50 & 0,0017 \\
\hline Unidentified & NA & NA & NA & NA & Present Clim. & 2,39 & 0,0032 \\
\hline Unidentified & NA & NA & NA & NA & Warm Clim. & 2,20 & 0,0016 \\
\hline Unidentified & NA & NA & NA & NA & Warm Clim. & 3,14 & 0,0007 \\
\hline Unidentified & NA & NA & NA & NA & Warm Clim. & 2,18 & 0,0023 \\
\hline Unidentified & NA & NA & NA & NA & Warm Clim. & 2,66 & 0,0007 \\
\hline Unidentified & NA & NA & NA & NA & Warm Clim. & 2,21 & 0,0034 \\
\hline
\end{tabular}


Table 11: Short-term impact of climatic conditions, lizard sex and bacterial richness at the beginning of the experiment on individual gut bacterial richness after 2 months of climatic treatment (total number of OTUs). Models were derived from a global model including climatic treatment (present versus warm conditions), initial bacterial richness (centred and scaled), interaction between initial bacterial richness and climatic treatment, and sex plus enclosure identity as a random intercept. The top best models $(\triangle \mathrm{AICc}<2)$ were then used in a model averaging method following Grueber et al (2011). $\mathrm{N}=48$ lizards. Symbols denote different levels of significance of the p-values: $* * * p<0.001, * * p<0.01, * p<0.05, . p<$ 0.10 .

\begin{tabular}{lllll}
\hline Parameter & Estimate & SE & p-value & $\begin{array}{l}\text { Relative } \\
\text { Importance }\end{array}$ \\
\hline $\begin{array}{l}\text { Climate } \\
\begin{array}{l}\text { Initial bacterial } \\
\text { richness }\end{array}\end{array}$ & -127.5 & 55.6 & $0.026^{*}$ & 0.63 \\
\hline
\end{tabular}


Table 12: Short-term impact of climatic conditions and lizard sex on the proportion of new OTUs and extinctions rates after 2 months of climatic treatment. Proportion of new OTUs is the number of OTUs present in September but not in May divided by the total number of OTUs present in September, and extinction rate is the number of OTUs present in May but absent in September divided by the total number of OTUs present in May. Models were derived from a global model including climatic treatment and sex plus random enclosure identity. The top best models $(\triangle \mathrm{AICc}<2)$ were used in a model averaging method following Grueber et al (2011). For the proportion of new OTUs, the top best model which did not include climate was largely better than the second best model $(\triangle \mathrm{AICc}>2)$ hence there was no need for model averaging. $\mathrm{N}=48$ lizards. Symbols denote different levels of significance of the p-values: $* * * \mathrm{p}<0.001, * * \mathrm{p}<0.01, * \mathrm{p}<0.05, . \mathrm{p}<0.10$.

\begin{tabular}{llllll}
\hline & Parameter & Estimate & SE & p-value & $\begin{array}{l}\text { Relative } \\
\text { importance }\end{array}$ \\
\hline Proportion of new OTUs & & & & & \\
& Sex & -0.04 & 0.02 & $0.02 *$ & - \\
Proportion of extinctions & & & & & \\
& Climate & 0.04 & 0.01 & $0.02 *$ & 1 \\
& Sex & -0.03 & 0.02 & 0.05 & 0.67 \\
\hline
\end{tabular}


Table 13: Pathways impact of climatic conditions on individual gut bacteria richness (total number of OTUs) through individual condition (body mass), social context (lizard density), sexual context (number of sexual partners) and potential dietary context (number of insect and arachnid families present in the enclosures). Summary of the averaged best models ( $\triangle \mathrm{AICc}<$ 2). Multicollinearity was not an issue as calculating Variance Inflation Factors (VIF) for each variable gave VIF always $<3$ (Supplementary Table 14). N = 150 lizards. Symbols denote different levels of significance of the p-values: *** p $<0.001, * * \mathrm{p}<0.01, * \mathrm{p}<0.05, \mathrm{p}<$ 0.10 .

\begin{tabular}{lllll}
\hline Parameter & Estimate & SE & p-value & $\begin{array}{l}\text { Relative } \\
\text { abundance }\end{array}$ \\
\hline Climate & -0.26 & 0.13 & $0.038^{*}$ & 0.74 \\
Lizard density & 0.22 & 0.12 & 0.077. & 0.66 \\
Number of insect families & 0.14 & 0.13 & 0.263 & 0.25 \\
Number of arachnid families & -0.08 & 0.11 & 0.462 & 0.11 \\
\hline
\end{tabular}


Table 14: List of the Variance Inflation Factors (VIF) calculated for each covariate from the models investigating identified bacterial richness as a function of climatic treatment, treatment, lizard density, number of sexual partners, body mass, number of insect families and number of arachnid families (Supplementary Table 13). VIF were calculated on the standardized variates following Grueber et al (2011). Variance inflation factors > 3 indicate potential collinearity problems (Grueber et al., 2011).

\begin{tabular}{ll}
\hline Variable & GVIF \\
\hline Temperature treatment & 2.12 \\
Density & 1.56 \\
Body Mass & 1.64 \\
Partners & 1.53 \\
Insects & 1.68 \\
Arachnids & 1.24 \\
\hline
\end{tabular}


Table 15: Impact of climatic conditions, lizard age and sex-classes on the predicted number of genes involved in the 4 main functions groups of bacteria selected using Picrust. Models were derived from a global model including climatic treatment, age and sex and every interactions as a covariate and enclosure identity as a random intercept. The top best models $(\triangle \mathrm{AICc}<2)$ were then used in a model averaging method following Grueber et al (2011). Sex estimates are given for males. Symbols denote different levels of significance of the p-values: $* * * \mathrm{p}<0.001, * * \mathrm{p}<0.01, * \mathrm{p}<0.05, . \mathrm{p}<0.10$.

\section{Metabolism}

\begin{tabular}{lllll}
\hline & & & & Relative \\
Parameter & Estimate & SE & p-value & Importance \\
\hline Climate & -0.14 & 0.11 & 0.20 & 1.00 \\
Sex & -15.14 & 3.78 & $<0.001 * * * *$ & 1.00 \\
Age & -0.44 & 0.52 & 0.40 & 0.32 \\
Climate*Sex & 0.42 & 0.12 & $<0.001 * * *$ & 1.00 \\
\hline
\end{tabular}

Environmental_Information_Processing

\begin{tabular}{lllll}
\hline Parameter & Estimate & SE & p-value & $\begin{array}{l}\text { Relative } \\
\text { Importance }\end{array}$ \\
\hline Climate & 0.18 & 0.08 & $0.02 *$ & 1.00 \\
Sex & 13.15 & 2.90 & $<0.001 * * *$ & 1.00 \\
Age & -0.61 & 2.69 & 0.82 & 0.79 \\
Climate*Sex & -0.39 & 0.09 & $<0.001 * * *$ & 1.00 \\
Age*Sex & -1.01 & 0.76 & 0.19 & 0.35 \\
Climate*Age & 0.12 & 0.09 & 0.21 & 0.31 \\
\hline
\end{tabular}

Genetic_Information_Processing

\begin{tabular}{lllll}
\hline & & & & Relative \\
Parameter & Estimate & SE & p-value & Importance \\
\hline Climate & -0.05 & 0.04 & 0.27 & 0.49 \\
Sex & -1.23 & 2.08 & 0.56 & 1.00 \\
Age & 1.62 & 1.87 & 0.39 & 0.30 \\
Climate*Sex & 0.11 & 0.05 & $0.04 *$ & 0.49 \\
Climate*Age & -0.09 & 0.05 & 0.10 & 0.16 \\
\hline
\end{tabular}

\section{Cellular Processes}

\begin{tabular}{lllll}
\hline & & & & Relative \\
Parameter & Estimate & SE & p-value & Importance \\
\hline- & - & - & - & - \\
\hline
\end{tabular}


Table 16: Impact of the interaction between climatic conditions and sex-classes on the 22 specific functions relevant for bacteria and selected using Picrust. Models were derived from a global model including climatic treatment, age and sex and every interactions as a covariate and enclosure identity as a random intercept. Given the strong sex-dependency of climatic effects, we only show the interaction between sex and climatic conditions after insuring that there was no simple effect of climatic conditions. Sex estimates are given for males. Our significance threshold for global analysis is 0.0022 according to Bonferroni corrections (denotes by a *, while a $\uparrow$ denotes a nearly significant effect). When significant, we explore this interaction using statistical analyses for each sex separately.

\begin{tabular}{|c|c|cc|cc|}
\hline & & \multicolumn{2}{|c|}{ Climate*Sex } & \multicolumn{2}{c|}{ Climate effect } \\
\hline Group & Function & Chi-sq & p-value & Females & Males \\
\hline \multirow{2}{*}{ Cellular } & Cell Growth and Death & 10.89 & $0.0009^{*}$ & Chi-sq $=8.74, \mathrm{p}=0.003$ & Chi-sq $=1.22, \mathrm{p}=0.270$ \\
processes & Cell Motility & 3.70 & 0.0544 & - & - \\
& Transport and Catabolism & 12.62 & $0.0003^{*}$ & Chi-sq $=4.10, \mathrm{p}=0.042 \quad$ Chi-sq $=6.34, \mathrm{p}=0.012$ \\
\hline Environmental & Membrane transport & 18.33 & $<0.0001^{*}$ & Chi-sq $=11.10, \mathrm{p}<0.001 \quad$ Chi-sq $=5.64, \mathrm{p}=0.018$ \\
Information & Signal Transduction & 5.72 & 0.0164 & - & - \\
Processing & Signaling Molecules and Interaction & 4.77 & 0.0294 & - & - \\
\hline \multirow{2}{*}{ Genetic } & Folding, Sorting and Degradation & 9.36 & $0.0022^{*}$ & Chi-sq $=3.85, \mathrm{p}=0.049$ & Chi-sq $=5.52, \mathrm{p}=0.019$ \\
Information & Replication and Repair & 7.90 & $0.0049 \dagger$ & Chi-sq $=4.16, \mathrm{p}=0.042 \quad$ Chi-sq $=2.77, \mathrm{p}=0.096$ \\
Processing & Transcription & 13.78 & $0.0002^{*}$ & Chi-sq $=2.12, \mathrm{p}=0.146 \quad$ Chi-sq $=7.00, \mathrm{p}=0.008$ \\
& Translation & 5.36 & 0.0261 & - & - \\
\hline
\end{tabular}


Table 16b

\begin{tabular}{|c|c|c|c|c|c|}
\hline \multirow[b]{2}{*}{ Group } & \multirow[b]{2}{*}{ Function } & \multicolumn{2}{|c|}{ Climate*Sex } & \multicolumn{2}{|c|}{ Climate effect } \\
\hline & & Chi-sq & p-value & Females & Males \\
\hline \multirow{12}{*}{ Metabolism } & Metabolism of Cofactors and Vitamins & 17.49 & $<0.0001 *$ & Chi-sq $=6.32, p=0.012$ & Chi-sq $=10.20, p=0.001$ \\
\hline & Metabolism of Other Amino Acids & 1.79 & 0.1897 & $\begin{array}{lll}1 & - \\
-1 & -1\end{array}$ & - \\
\hline & Metabolism of Terpenoids and Polyketides & 7.39 & $0.0066 \dagger$ & Chi-sq $=0.56, p=0.453$ & Chi-sq $=3.82, p=0.051$ \\
\hline & Nucleotide Metabolism & 4.45 & 0.0349 & - & - \\
\hline & Xenobiotics Biodegradation and Metabolism & 2.26 & 0.1327 & - & - \\
\hline & Amino Acid Metabolism & 14.03 & $0.0002 *$ & Chi-sq $=3.72, p=0.053$ & Chi-sq $=4.72, p=0.029$ \\
\hline & Biosynthesis of Other Secondary Metabolites & 4.48 & 0.0342 & - & - \\
\hline & Carbohydrate Metabolism & 0.22 & 0.6360 & - & - \\
\hline & Energy Metabolism & 20.21 & $<0.0001 *$ & Chi-sq $=11.46, \mathrm{p}<0.001$ & Chi-sq $=5.17, p=0.023$ \\
\hline & Enzyme Families & 1.63 & 0.2010 & - & - \\
\hline & Glycan Biosynthesis and Metabolism & 0.18 & 0.6734 & - & - \\
\hline & Lipid Metabolism & 8.04 & $0.0046 \dagger$ & Chi-sq $=0.47, p=0.492$ & Chi-sq $=4.89, p=0.027$ \\
\hline
\end{tabular}


Table 17: Impact of bacterial richness (total number of OTUs) on lizard subsequent survival in a common garden experiment $(\mathrm{N}=78$ lizards). Models were derived from a global model including total bacterial richness (centred and scaled) climatic treatment the year before, age, sex and lizard body condition (residuals of a linear model of body mass by body length, centred and scaled) to account for differences in individual quality, plus enclosure identity as a random intercept. The top best models $(\triangle \mathrm{AICc}<2)$ were then used in a model averaging method following Grueber et al (2011). Multicollinearity was not an issue as calculating Variance Inflation Factors (VIF) for each variable gave VIF always < 3 (Supplementary Table 18). Symbols denote different levels of significance of the p-values: $* * * p<0.001$, $* * p<$ $0.01, * \mathrm{p}<0.05, \mathrm{p}<0.10$.

\begin{tabular}{lllll}
\hline Parameter & Estimate & SE & p-value & $\begin{array}{l}\text { Relative } \\
\text { abundance }\end{array}$ \\
\hline Bacterial richness & 0.70 & 0.34 & $0.04 *$ & 1 \\
Climatic treatment the year before & 0.16 & 0.07 & $0.02 *$ & 1 \\
Sex & 0.58 & 0.53 & 0.28 & 0.29 \\
Body Condition & 0.20 & 0.27 & 0.46 & 0.21 \\
\hline
\end{tabular}


Table 18: List of the Variance Inflation Factors (VIF) calculated for each covariate from the models investigating lizard survival as a function of total bacterial richness (standardized) climatic treatment the year before, age, sex and lizard body condition (standardized), see Supplementary Table 17. VIF were calculated on the standardized variates following Grueber et al (2011). Variance inflation factors > 3 indicate potential collinearity problems (Grueber et al., 2011).

\begin{tabular}{ll}
\hline Variable & GVIF \\
\hline Bacterial richness & 1.13 \\
Climatic treatment the year before & 1.16 \\
Age & 1.16 \\
Sex & 1.20 \\
Body Condition & 1.37 \\
\hline
\end{tabular}


Table 19: Component loading of Axis 1 from a PCA with 49 OTUs differentially expressed in present and warm climates

\begin{tabular}{|c|c|c|c|}
\hline & & 1 & \\
\hline 1 & & & 1 \\
\hline & & & \\
\hline & & & \\
\hline & & & \\
\hline & & & \\
\hline 264 & & & \\
\hline 67 & & & \\
\hline 78 & & & \\
\hline & & & \\
\hline 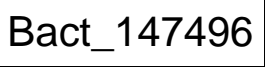 & & & \\
\hline 46 & & & \\
\hline 027 & & & \\
\hline & & & \\
\hline & & & \\
\hline & & & \\
\hline 9 & & & \\
\hline 3 & & & \\
\hline 22 & esent & & \\
\hline & & & \\
\hline & & & \\
\hline & & & \\
\hline 37 & & & 2.2 \\
\hline Dave & & & \\
\hline Dace & & & \\
\hline Bact & & & \\
\hline & & & \\
\hline $\mathrm{Ba}$ & $\mathrm{nt}$ & & \\
\hline$B$ & & & \\
\hline 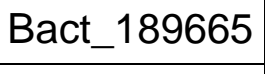 & & & \\
\hline & & & \\
\hline act_191378 & Present & 0.2081549 & $1.058522 \mathrm{e}-02$ \\
\hline
\end{tabular}




\begin{tabular}{|c|c|c|c|}
\hline OTUs & Climate & correlation & p.value \\
\hline Bact_147870 & Warm & -0.1897994 & $2.000257 \mathrm{e}-02$ \\
\hline Bact_200631 & Warm & -0.1947835 & $1.691414 \mathrm{e}-02$ \\
\hline Bact_074132 & Warm & -0.2127520 & $8.952385 \mathrm{e}-03$ \\
\hline Bact_200355 & Warm & -0.2567498 & $1.516200 \mathrm{e}-03$ \\
\hline Bact_084667 & Warm & -0.2899617 & $3.191692 \mathrm{e}-04$ \\
\hline Bact_147522 & Warm & -0.2961281 & $2.338307 \mathrm{e}-04$ \\
\hline Bact_168890 & Warm & -0.3198056 & $6.626288 \mathrm{e}-05$ \\
\hline Bact_158110 & Warm & -0.4399554 & $1.768642 \mathrm{e}-08$ \\
\hline Bact_200283 & Warm & -0.4404621 & $1.695848 \mathrm{e}-08$ \\
\hline Bact_031673 & Warm & -0.4854261 & $3.055819 \mathrm{e}-10$ \\
\hline Bact_136975 & Warm & -0.5108520 & $2.403995 \mathrm{e}-11$ \\
\hline Bact_010574 & Warm & -0.5446106 & $5.838705 \mathrm{e}-13$ \\
\hline Bact_115913 & Warm & -0.5666569 & $4.078131 \mathrm{e}-14$ \\
\hline
\end{tabular}


Table 20: Impact of bacterial OTUs different between climates, summarized using a Principal component analysis (Bacterial axis 1), on lizard subsequent survival in a common garden experiment $(\mathrm{N}=78$ lizards). Models were derived from a global model including this axis (centred and scaled), climatic treatment the year before, age, sex and lizard body condition (residuals of a linear model of body mass by body length, centred and scaled) to account for differences in individual quality, plus enclosure identity as a random intercept. The top best models $(\triangle \mathrm{AICc}<2)$ were then used in a model averaging method following Grueber et al (2011). Symbols denote different levels of significance of the p-values: $* * * p<0.001$, ** $\mathrm{p}<$ $0.01, * \mathrm{p}<0.05, \mathrm{p}<0.10$

\begin{tabular}{lllll}
\hline Parameter & Estimate & SE & p-value & $\begin{array}{l}\text { Relative } \\
\text { abundance }\end{array}$ \\
\hline Bacterial axis 1 & 0.78 & 0.35 & $0.01 *$ & 1 \\
Climatic treatment the year before & 0.19 & 0.08 & $0.01 *$ & 1 \\
Sex & 0.45 & 0.53 & 0.49 & 0.25 \\
Body Condition & 0.17 & 0.27 & 0.52 & 0.21 \\
\hline
\end{tabular}

